See discussions, stats, and author profiles for this publication at: https://www.researchgate.net/publication/332268894

\title{
A FRACTIONAL STEP METHOD FOR COMPUTATIONAL AEROACOUSTICS USING WEAK IMPOSITION OF DIRICHLET BOUNDARY CONDITIONS
}

\section{Preprint·April 2019}

CITATIONS

0

3 authors:

\section{Samuel Parada}

Universitat Politècnica de Catalunya

2 PUBLICATIONS 0 CITATIONS

SEE PROFILE

\section{Ramon Codina}

Universitat Politècnica de Catalunya

253 PUBLICATIONS 6,818 CITATIONS

SEE PROFILE
READS

212

Joan Baiges

Universitat Politècnica de Catalunya

62 PUBLICATIONS 502 CITATIONS

SEE PROFILE

Some of the authors of this publication are also working on these related projects:

Project Design and analysis of numerical methods for viscoelastic flows View project

Project Topology optimization in fluid-structure interaction problems (TOP-FSI) View project 


\title{
A FRACTIONAL STEP METHOD FOR COMPUTATIONAL AEROACOUSTICS USING WEAK IMPOSITION OF DIRICHLET BOUNDARY CONDITIONS
}

\author{
SAMUEL PARADA JOAN BAIGES $^{\ddagger}$ AND RAMON CODINA $^{\S, \ddagger}$
}

\begin{abstract}
In this work we consider the approximation of the isentropic Navier-Stokes equations. The model we present is capable of taking into account acoustics and flow scales at once. Once space and time discretizations have been chosen, it is very convenient from the computational point of view to design splitting schemes in time so as to permit a segregated calculation of the problem unknowns. While these splitting or fractional step schemes are well established for incompressible flows, much less is known in the case of isentropic flows. We discuss this issue in this article and, furthermore, we study the way to impose Dirichlet boundary conditions weakly via Nitsche's method. In order to avoid spurious reflections of the acoustic waves, Nitsche's method is combined with a non-reflecting boundary condition. Employing a purely algebraic approach to discuss the problem, some of the boundary contributions are treated explicitly and we explain how these are included in the different steps of the final algorithm. Numerical evidence shows that this explicit treatment does not have a significant impact on the convergence rate of the resulting time integration scheme. The equations of the formulation are solved using a subgrid scale technique based on a term-by-term stabilization.
\end{abstract}

Keywords: Isentropic flow, fractional step methods, weak boundary conditions, term-byterm stabilization, aeroacoustics

\section{INTRODUCTION}

Within the field of Computational Aeroacoustics (CAA), the solution of the complete set of Navier-Stokes equations written in its conservative form, i.e. the coupled problem involving mass, momentum and energy conservation equations, is referred as Direct Noise Computation (DNC) 11. This formulation represents a direct path to consistently deal with aerodynamic and acoustic scales in an unified manner. The solution of this fully compressible problem via the Finite Element Method (FEM) [2] is known to be excessively demanding in terms of computational power. Likewise, most of the compressible flow solvers found in the literature exhibit an inadequate performance in the low Mach number regime. This is mainly due to the fact that flow and acoustic scales start to considerably differ one from each other under the subsonic condition as the Mach number is progressively reduced. As a consequence, the algebraic systems arising from those formulations are usually ill-conditioned.

With the aim of overcoming such conditioning issues, several hybrid methods arose for which the computations of aerodynamics and acoustics are decoupled. See e.g. 3, 4, for the socalled incompressible-acoustic split method. The most remarkable work in this area is probably the well-known Lighthill analogy [5], in which the acoustic field is obtained upon the derivation of a source term computed with the flow equations. Although these methods allow a certain flexibility, in the sense that they permit the usage of different models for

Date: March 26, 2019.

‡ Universitat Politècnica de Catalunya, Barcelona Tech, Jordi Girona 1-3, Edifici C1, 08034 Barcelona, Spain.

$\S$ Centre Internacional de Mètodes Numèrics en Enginyeria (CIMNE), Edifici C1, Campus Nord UPC, Gran Capità s/n, 08034 Barcelona, Spain.

E-mails: sparada@cimne.upc.edu (SP), joan.baiges@upc.edu (JB), ramon.codina@upc.edu (RC). 
flow and acoustics, their approximation errors still need to be properly analyzed, as they assume only a one-way coupling from flow to acoustics and acoustic terms need to be correctly derived.

The formulation presented in this work, recently published in [6], aims at combining the simplicity of hybrid methods with the unified scale computation of DNC. We will see that this methodology can be understood as an extension of the incompressible flow equations, since the final problem to be solved requires to compute only velocity and pressure fields, being the thermal problem mathematically uncoupled. This is possible due to the fact that the entropy remains constant, which in turn allows one to establish a connection between density and pressure perturbations, assuming a low Mach number flow, with neither shocks nor thermal sources. The computational cost of the present technique is reduced with respect to other methods since, apart from the fact that both acoustic and flow scales are solved altogether and that we get rid of the energy conservation equation, the final system is better conditioned (we refer to [6] for a detailed discussion). For simplicity in the exposition, the ideal gas law has been assumed, yet other equations of state may be possible.

Solving monolithically the algebraic system of equations that arises after the discretization of the continuous isentropic problem is the classical strategy. Despite of the several simplifications that can be introduced thanks to the constant entropy assumption, solving the resulting linear system of equations might still be computationally expensive, specially in 3D geometries. The unknowns are highly coupled and nonlinearities need to be solved too. An alternative to the standard approach is to solve the problem by means of a fractional step method in time. This technique consists in segregating the calculation of the unknowns, so that they can be computed separately, probably with the addition of some correction steps. On the negative side, fractional step methods have an associated temporal error, frequently labeled as splitting or fractional error. It is indispensable to ensure that such error is at least of the order of the integration scheme used in time, with the purpose of maintaining the global temporal accuracy of the method.

Fractional step methods were originally called projection methods (indistinctly called fractional step or segregation methods in the following) as they were based on the decomposition of differential operators at the continuous level. The pioneering works of Chorin and Temam [7, 8] in the late 1960's established the basis of this novel technique. Apart from this continuous approach, fractional step methods can be introduced at the purely algebraic level too, which is the methodology adopted in this work. For a review on both approaches in the case of the incompressible Navier-Stokes equations, we refer the reader to [9, 10]. Eventually, fractional step methods have enjoyed an extensive recognition mainly due to two reasons: they allow an important reduction of computational time and present an intrinsic stability over the pressure gradient term [11].

Apart from a possible compatibility restriction between velocity and pressure interpolating spaces, the convective terms appearing in the governing equations may render the solution unstable when using a finite element formulation, showing spurious nodeto-node oscillations. Though this unstable behavior could be avoided setting a specific mesh size (which commonly is not computationally affordable), stabilized formulations appear to circumvent this issue. In these formulations, the weak form of the problem obtained by the classical Galerkin method is modified upon the introduction of some mesh-dependent terms weighted by the residuals (or even part of them) of the differential equations. Several numerical techniques have been developed within this context: the well known Streamline/Upwind-Petrov-Galerkin (SUPG) method [12] or Galerkin-Least Square (GLS) method [13], the Taylor-Galerkin method [14] and the Variational MultiScale method (VMS) [15, 16], being the latter the one adopted in this work. The VMS technique provides a general variational framework for subgrid scale models [17]. 
The key idea behind the VMS approach is to split the unknowns of the problem into two scales, namely, the scale that can be approximated by the finite element mesh and the subgrid scale, the unresolvable one. The general methodology consists in finding an approximation for the subgrid scale so as to yield a stable formulation involving only the finite element scales, hence maintaining the number of degrees of freedom of the starting Galerkin variational problem. There are different ways to model the subgrid scale, provided a definition of the functional space where it belongs. In this article, we will define such space as the orthogonal one to the finite element space and, using this concept, we will state a term-by-term technique by neglecting the extra cross products which do not play any stability role in the formulation. We will see that this formulation is not residual based, and hence not consistent, being consistency understood in the classical finite element context. However, for the incompressible flow problem this term-by-term possibility provides a slightly improved pressure stability [18], and in [19, 20] it was applied to the viscoelastic flow problem making it possible to solve more elastic cases than a residual-based formulation.

As for any other compressible formulation, the treatment of waves at the external boundaries is a problematic issue that needs to be faced. Solvers for compressible flows need to be able to let acoustic waves leave the domain without any deceptive reflection. This distinguishing feature has already been studied in the past and as a result, there are several numerical techniques which deal with the backscattering of waves; see for instance the review in [21, 22] and references therein. At Dirichlet boundaries, where the velocity is to be prescribed, there must be a compatibility requirement between the treatment of acoustic waves and the flow boundary conditions. This fact is even more significant taking into account that the formulation we propose solves aerodynamic and acoustic scales simultaneously. In this sense, a novel method for the unified prescription of boundary conditions for the isentropic problem was introduced in [6] (Section 3), which is based on a combination of a weak imposition of Dirichlet boundary conditions [23] of the mean flow variables, plus a Sommerfeld non-reflecting boundary condition for the acoustic component of the pressure 24]. We will formulate here its segregated counterpart, solving the problem in a split manner free of artificial wave reflections.

The article is organized as follows: in Section 2 the isentropic compressible NavierStokes equations are introduced, as well as its variational formulation. The details of the compatible prescription of boundary conditions are reviewed in Section 3, whereas in Section 4 we present the variational formulation and the monolithic time discretization of the problem with the boundary conditions described earlier. Section 5 is devoted to the design of the second order fractional step scheme from an algebraic viewpoint, taking into account the modifications due to the application of boundary conditions. In Section 6. we state the stabilized finite element formulation we favor, together with the relevant adjustments that need to be considered in the prior fractional step algorithm. Numerical experiments are conducted in Section 7 and, finally, conclusions are drawn in Section 8 .

\section{ISENTROPIC COMPRESSIBLE FLOW PROBLEM}

2.1. Initial considerations. Let us start the exposition of our work by recalling some of the basic relations of the compressible flow theory for ideal gases, (see e.g. [25] for details). By definition of an isentropic flow, the entropy remains constant. This fact allows one to show that pressure and density are related through the following fundamental expression,

$$
\frac{p}{\rho^{\gamma}}=C \quad \text { (a constant), }
$$

where $\gamma$ is the socalled adiabatic coefficient, $\gamma \doteq c_{p} / c_{v}$, being $c_{p}$ and $c_{v}$ the specific heat of the fluid at constant pressure and volume, respectively. Additionally, $p$ denotes the total pressure and $\rho$ is the total density, including any probable perturbations that the compressible nature of the medium might cause. There exist two useful expressions which relate 
density and pressure for two locations, being one of them at the stagnation conditions, that is to say, those that would exist if the flow at any point of a stream was isentropically brought to rest,

$$
\begin{aligned}
& \frac{\rho_{0}}{\rho}=\left(1+\frac{\gamma-1}{2} \mathrm{Ma}^{2}\right)^{\frac{1}{\gamma-1}}, \\
& \frac{p_{0}}{p}=\left(1+\frac{\gamma-1}{2} \mathrm{Ma}^{2}\right)^{\frac{\gamma}{\gamma-1}},
\end{aligned}
$$

where $(\cdot)_{0}$ stands for variables at stagnation conditions. The symbol Ma refers to the Mach number, defined as $\mathrm{Ma} \doteq|\boldsymbol{u}| / c_{0}$ being $|\boldsymbol{u}|$ the modulus of the pointwise flow velocity and $c_{0}$ the speed of sound of an ideal gas, computed as $c_{0} \doteq \sqrt{\gamma R \theta_{0} / \mathcal{M}}$. In this last expression, $R[\mathrm{~J} / \mathrm{K}-\mathrm{mol}]$ is the universal gas constant, $\mathcal{M}[\mathrm{kg} / \mathrm{mol}]$ is the molar mass of the gas under consideration and $\theta_{0}[\mathrm{~K}]$ denotes the temperature.

Relating (2)-(3) one can easily derive the following equality,

$$
\frac{p_{0}}{p}=\left(\frac{\rho_{0}}{\rho}\right)^{\gamma},
$$

which is in direct concordance with the fundamental expression of isentropic flows (1).

Now, taking derivatives with respect to time in both sides of (4) and recalling the equation of state of an ideal gas, i.e., $p_{0} \mathcal{M}=\rho_{0} R \theta_{0}$, an expression directly relating density and pressure time derivatives arises,

$$
\frac{\partial p}{\partial t}=\frac{p_{0}}{\rho_{0}} \gamma\left(1+\frac{\gamma-1}{2} \mathrm{Ma}^{2}\right)^{-1} \frac{\partial \rho}{\partial t}=\frac{R \theta_{0}}{\mathcal{M}} \gamma\left(1+\frac{\gamma-1}{2} \mathrm{Ma}^{2}\right)^{-1} \frac{\partial \rho}{\partial t} .
$$

If instead of the time derivative, one takes spatial derivatives, an equivalent expression can be found relating gradients, and both equations can be simplified if we identify the speed of sound as,

$$
c=c_{0}\left(1+\frac{\gamma-1}{2} \mathrm{Ma}^{2}\right)^{-\frac{1}{2}},
$$

so that in practice the following relations can be used,

$$
\frac{\partial p}{\partial t}=c^{2} \frac{\partial \rho}{\partial t} \quad ; \quad \nabla p=c^{2} \nabla \rho
$$

Equation (6) establishes a connection between pressure and density variations in a straightforward manner allowing one to considerably reduce the general complexity of the compressible Navier-Stokes problem while making possible to capture the acoustic scales of the flow. This is in contraposition with other non-isentropic formulations for low Mach flows (see e.g. [26]) in which density variations are related to temperature instead of pressure, and hence no acoustics are modeled.

2.2. Initial and boundary value problem. The problem we analyze in this work consists in the mass and momentum conservation equations defined during a time interval $(0, T)$ and over an open and bounded physical domain $\Omega \subset \mathbb{R}^{d}$, being $d=2,3$ the space dimension. Considering a Newtonian fluid under isentropic compressible conditions, the problem is initially written as,

$$
\begin{aligned}
\rho \frac{\partial \boldsymbol{u}}{\partial t}+\rho(\boldsymbol{u} \cdot \boldsymbol{\nabla}) \boldsymbol{u}-\mu \boldsymbol{\Delta} \boldsymbol{u}-\frac{\mu}{3} \boldsymbol{\nabla}(\boldsymbol{\nabla} \cdot \boldsymbol{u})+\boldsymbol{\nabla} p=\boldsymbol{f} & \text { in } \Omega \times(0, T), \\
\frac{\partial \rho}{\partial t}+\boldsymbol{u} \cdot \boldsymbol{\nabla} \rho+\rho \boldsymbol{\nabla} \cdot \boldsymbol{u}=0 & \text { in } \Omega \times(0, T),
\end{aligned}
$$

where $\rho$ is the density, $p$ is the pressure, $\boldsymbol{u}$ stands for the velocity field and $\mu$ for the fluid dynamic viscosity, and $\boldsymbol{f}$ is a given force vector. As usual, bold characters refer 
to vector variables and the symbol $\boldsymbol{\Delta}(\cdot)$ denotes the Laplacian operator. Making use of (6), the continuity equation can be reformulated so that the strong form of the isentropic compressible Navier-Stokes governing equations finally reads,

$$
\begin{aligned}
\rho \frac{\partial \boldsymbol{u}}{\partial t}+\rho(\boldsymbol{u} \cdot \boldsymbol{\nabla}) \boldsymbol{u}-\mu \boldsymbol{\Delta} \boldsymbol{u}-\frac{\mu}{3} \boldsymbol{\nabla}(\boldsymbol{\nabla} \cdot \boldsymbol{u})+\nabla p=\boldsymbol{f} & \text { in } \Omega \times(0, T), \\
\frac{1}{\rho c^{2}} \frac{\partial p}{\partial t}+\frac{1}{\rho c^{2}} \boldsymbol{u} \cdot \boldsymbol{\nabla} p+\boldsymbol{\nabla} \cdot \boldsymbol{u}=0 & \text { in } \Omega \times(0, T),
\end{aligned}
$$

where the sound velocity $c$ can be computed using (5). Let us rewrite the problem in a more compact manner. If we set $\boldsymbol{U} \doteq[\boldsymbol{u}, p]^{t}, \mathcal{F}=[\boldsymbol{f}, 0]^{t}$ and define

$$
\mathcal{L}(\boldsymbol{a} ; \boldsymbol{U}) \doteq\left[\rho(\boldsymbol{a} \cdot \boldsymbol{\nabla}) \boldsymbol{u}-\mu \Delta \boldsymbol{u}-\frac{\mu}{3} \boldsymbol{\nabla}(\boldsymbol{\nabla} \cdot \boldsymbol{u})+\nabla p, \frac{1}{\rho c^{2}} \boldsymbol{a} \cdot \boldsymbol{\nabla} p+\boldsymbol{\nabla} \cdot \boldsymbol{u}\right]^{t},
$$

for some advection velocity $\boldsymbol{a}$ and,

$$
\mathcal{D}_{t}(\boldsymbol{U}) \doteq\left[\rho \frac{\partial \boldsymbol{u}}{\partial t}, \frac{1}{\rho c^{2}} \frac{\partial p}{\partial t}\right]^{t}
$$

we may state problem (7)-(8) as,

$$
\mathcal{D}_{t}(\boldsymbol{U})+\mathcal{L}(\boldsymbol{u}, \boldsymbol{U})=\mathcal{F} .
$$

The governing equations need to be complemented with a suitable set of both initial and boundary conditions to ensure the well-posedness of the problem, being the latter discussed in the next section. The initial conditions are set for velocity and pressure, and shall be written in the form $\boldsymbol{u}=\boldsymbol{u}^{0}$ and $p=p^{0}$ at time $t=0$, being $\boldsymbol{u}^{0}$ and $p^{0}$ functions defined over the whole domain $\Omega$. Note that problem $(7)-(8)$ can be seen as a direct extension of the incompressible Navier-Stokes case with the addition of a temporal and convective term for the pressure.

The reader might have noticed that although several simplifications were introduced, the governing equations still depend on both density and sound velocity fields. In order to resolve these additional nonlinearities, Equations (2) and (5) are used to complete the formulation and computed explicitly in time, so that one would need to solve only for velocity and pressure fields, hence allowing to capture the acoustic scales.

2.3. Variational form. Let us first introduce some notation. The space of square integrable functions in a domain $\omega$ will be denoted by $L^{2}(\omega)$. We shall also use the symbol $\langle\cdot, \cdot\rangle_{\omega}$ to refer to the integral of the product of two functions, assuming it is well defined. Subscript $\omega$ will be omitted when $\omega=\Omega$. In addition to this, for a given Banach space $\mathbb{X}$ we write as $L^{m}(0, T ; \mathbb{X})$ the Bochner spaces of functions such that their $\mathbb{X}$-norm is an $L^{m}(0, T)$ function in time, i.e., its $m$-th power is integrable if $1 \leq m<\infty$ or bounded if $m=\infty$. Finally, $\boldsymbol{n}$ is the geometric unit outward normal vector on the boundary $\Gamma=\partial \Omega$.

Let now $\mathbb{V}^{d}$ and $\mathbb{Q}$ be, respectively, the proper functional spaces where velocity and pressure solutions are well defined for each fixed time $t \in(0, T)$, with appropriate regularity not analyzed here. The weak form of the problem is derived by testing (7)-(8) against arbitrary test functions, namely $\boldsymbol{v} \in \mathbb{V}^{d}$ and $q \in \mathbb{Q}$. The variational problem prior to the application of boundary conditions written in a condensed manner reads: find $\boldsymbol{U} \in$ $L^{2}\left(0, T ; \mathbb{V}^{d} \times \mathbb{Q}\right)$ such that the initial conditions are satisfied and,

$$
\left\langle\mathcal{D}_{t}(\boldsymbol{U}), \boldsymbol{V}\right\rangle+\mathcal{A}(\boldsymbol{u} ; \boldsymbol{U}, \boldsymbol{V})=\langle\boldsymbol{v}, \boldsymbol{f}\rangle+\langle\boldsymbol{v}, \boldsymbol{n} \cdot \boldsymbol{\sigma}(\boldsymbol{u}, p)\rangle_{\Gamma},
$$

for all $\boldsymbol{V} \doteq[\boldsymbol{v}, q]^{t} \in \mathbb{V}^{d} \times \mathbb{Q}$ and where,

$$
\begin{aligned}
\mathcal{A}(\boldsymbol{u} ; \boldsymbol{U}, \boldsymbol{V}) & \doteq\langle\rho \boldsymbol{v},(\boldsymbol{u} \cdot \boldsymbol{\nabla}) \boldsymbol{u}\rangle+\mu\langle\nabla \boldsymbol{v}, \boldsymbol{\nabla} \boldsymbol{u}\rangle+\frac{\mu}{3}\langle\boldsymbol{\nabla} \cdot \boldsymbol{v}, \boldsymbol{\nabla} \cdot \boldsymbol{u}\rangle-\langle\boldsymbol{\nabla} \cdot \boldsymbol{v}, p\rangle \\
& +\left\langle q, \frac{1}{\rho c^{2}} \boldsymbol{u} \cdot \nabla p\right\rangle+\langle q, \boldsymbol{\nabla} \cdot \boldsymbol{u}\rangle .
\end{aligned}
$$


As usual, integration by parts was used and the stress tensor was identified as $\boldsymbol{\sigma}(\boldsymbol{u}, p)=$ $-p \boldsymbol{I}+\mu \boldsymbol{\nabla} \boldsymbol{u}+\frac{\mu}{3}(\boldsymbol{\nabla} \cdot \boldsymbol{u}) \boldsymbol{I}$. Special care needs to be taken on the imposition of boundary conditions of the isentropic problem. This is to be treated next, so that the right hand side boundary term of (9) is modified in order to allow a compatible treatment of waves and flow velocity conditions.

\section{IMPOSITION OF BOUNDARY CONDITIONS}

Since the formulation we present aims at accounting for both flow and acoustic scales at once, a special type of condition must be imposed for the pressure field, whose main purpose is to allow the sound waves to leave the external boundaries of the computational domain smoothly. In this section we review the method proposed in [6] for the prescription of boundary conditions for the isentropic problem.

3.1. Unknown and boundary splitting. The starting idea of the method is the splitting of the two unknown fields of the problem, i.e., velocity and pressure, into mean and oscillatory components. For a given time instant $t \in(0, T)$ and a point in the spatial domain $\boldsymbol{x} \in \Omega$, we have,

$$
\boldsymbol{u}(\boldsymbol{x}, t)=\overline{\boldsymbol{u}}(\boldsymbol{x}, t)+\boldsymbol{u}^{\prime}(\boldsymbol{x}, t), \quad p(\boldsymbol{x}, t)=\bar{p}(\boldsymbol{x}, t)+p^{\prime}(\boldsymbol{x}, t),
$$

where the mean variables are mathematically described as,

$$
\overline{\boldsymbol{u}}(\boldsymbol{x}, t) \doteq \frac{1}{T_{w}} \int_{t-T_{w}}^{t} \boldsymbol{u}(\boldsymbol{x}, s) d s, \quad \bar{p}(\boldsymbol{x}, t) \doteq \frac{1}{T_{w}} \int_{t-T_{w}}^{t} \bar{p}(\boldsymbol{x}, s) d s
$$

being $T_{w}$ an appropriate time window. In the following, we will identify the oscillatory components with the acoustic fluctuations and the mean variables with the flow variables. The main idea is that in (11), $T_{w}$ implicitly defines a filtering frequency for the acoustic waves.

Let us now introduce a boundary splitting at the continuous level, in order to manage the flow and acoustic boundary conditions in a suitable manner. The boundary $\Gamma=\partial \Omega$ is divided into three different disjoint subsets, namely, $\Gamma_{S}, \Gamma_{L}$ and $\Gamma_{O}$ (see Fig. 1). These subsets are such that $\Gamma_{S} \cap \Gamma_{L}=\emptyset, \Gamma_{L} \cap \Gamma_{O}=\emptyset, \Gamma_{O} \cap \Gamma_{S}=\emptyset$ and $\Gamma_{S} \cup \Gamma_{L} \cup \Gamma_{O}=\Gamma$. The boundary $\Gamma_{S}$ refers to the solid boundary and $\Gamma_{L}$ is identified with the lateral boundaries, i.e. any frontier with at least one component of the velocity prescribed to a known value. Finally, $\Gamma_{O}$ stands for the outlet. On $\Gamma_{L}$ and $\Gamma_{O}$, which are in the far field, it is assumed that the acoustic scales are dominant.

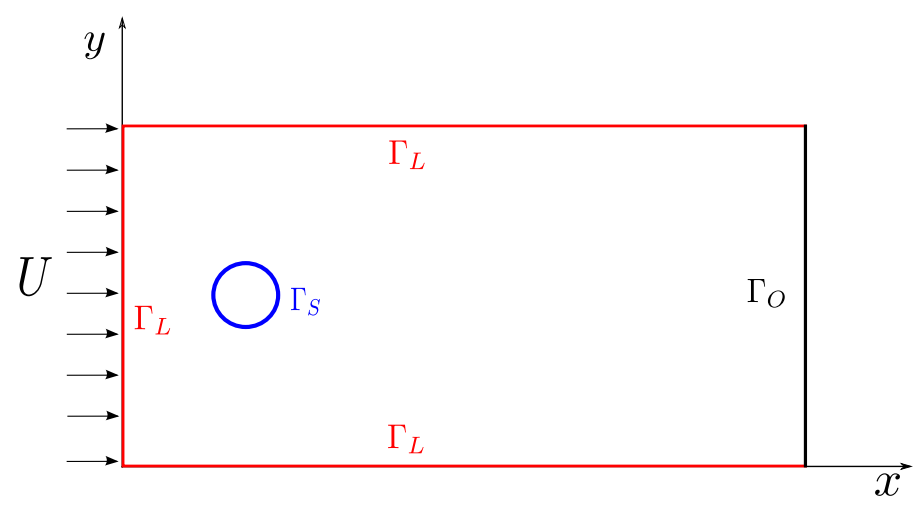

Figure 1. Example of boundary splitting at the continuous level to allow a compatible prescription of boundary conditions. This setting corresponds to the examples presented in Section 7. 
3.2. Unified prescription of boundary conditions. The two main ingredients of the methodology are: the weak prescription of essential boundary conditions and the application of Sommerfeld-like non-reflecting boundary conditions, NRBC. Next, we summarize the different conditions to be applied on each boundary.

On the solid boundary, i.e. $\Gamma_{S}$, the velocity is known and we apply:

$$
\boldsymbol{u}=\overline{\boldsymbol{u}}+\boldsymbol{u}^{\prime}=\boldsymbol{u}_{s} \quad \text { on } \quad \Gamma_{S},
$$

being $\boldsymbol{u}_{s}$ the prescribed velocity on the solid boundary. This is a classical strong-Dirichlet type boundary.

On the frontiers belonging to the truncation boundary $\Gamma_{L}$, distinct conditions are enforced:

- The mean value of the velocity is prescribed to the flow inlet velocity,

$$
\overline{\boldsymbol{u}}=\boldsymbol{u}_{L} \quad \text { on } \quad \Gamma_{L} .
$$

- A Sommerfeld-like non-reflecting boundary condition is prescribed for the acoustic component of the velocity field. In the normal direction to the boundary we define,

$$
\boldsymbol{n} \cdot \boldsymbol{u}^{\prime}=-\frac{1}{\rho c} \boldsymbol{n} \cdot\left[\boldsymbol{n} \cdot \boldsymbol{\sigma}\left(\boldsymbol{u}^{\prime}, p^{\prime}\right)\right] \quad \text { on } \quad \Gamma_{L},
$$

and for the tangential direction we directly write,

$$
\boldsymbol{m} \cdot\left[\boldsymbol{n} \cdot \boldsymbol{\sigma}\left(\boldsymbol{u}^{\prime}, p^{\prime}\right)\right]=0 \quad \text { on } \quad \Gamma_{L},
$$

for any vector $\boldsymbol{m}$ in the tangent direction to $\Gamma_{L}$.

The prescription of $\overline{\boldsymbol{u}}=\boldsymbol{u}_{L}$ will be done weakly through the popular Nitche's method [27] which provides a better conditioned problem. Finally, on the outflow boundary $\Gamma_{O}$, the following conditions are considered:

- The mean value tractions are prescribed to a value $\boldsymbol{t}_{O}$, i.e.,

$$
\boldsymbol{n} \cdot \boldsymbol{\sigma}(\overline{\boldsymbol{u}}, \bar{p})=\boldsymbol{t}_{O} \quad \text { on } \quad \Gamma_{O} .
$$

- The same approach as in $\Gamma_{L}$ is used now for the fluctuating component. Then,

$$
\begin{aligned}
& \boldsymbol{n} \cdot \boldsymbol{u}^{\prime}=-\frac{1}{\rho c} \boldsymbol{n} \cdot\left[\boldsymbol{n} \cdot \boldsymbol{\sigma}\left(\boldsymbol{u}^{\prime}, p^{\prime}\right)\right] \text { on } \Gamma_{O}, \\
& \boldsymbol{m} \cdot\left[\boldsymbol{n} \cdot \boldsymbol{\sigma}\left(\boldsymbol{u}^{\prime}, p^{\prime}\right)\right]=0 \quad \text { on } \Gamma_{O} .
\end{aligned}
$$

This is a boundary with natural conditions prescribed for $\overline{\boldsymbol{u}}$ and Sommerfeld conditions for $\boldsymbol{u}^{\prime}$.

Taking now into account these definitions, the prescription of boundary conditions in the weak form of the problem can be done upon the modification of the right hand side term of (9). Thus,

$$
\begin{aligned}
\langle\boldsymbol{v}, \boldsymbol{n} \cdot \boldsymbol{\sigma}(\boldsymbol{u}, p)\rangle_{\Gamma}= & \langle\boldsymbol{v}, \boldsymbol{n} \cdot \boldsymbol{\sigma}(\boldsymbol{u}, p)\rangle_{\Gamma_{L}}+\langle\boldsymbol{v}, \boldsymbol{n} \cdot \boldsymbol{\sigma}(\boldsymbol{u}, p)\rangle_{\Gamma_{O}} \\
= & \langle\boldsymbol{v}, \boldsymbol{n} \cdot \boldsymbol{\sigma}(\overline{\boldsymbol{u}}, \bar{p})\rangle_{\Gamma_{L}}+\left\langle\boldsymbol{v}, \boldsymbol{n} \cdot \boldsymbol{\sigma}\left(\boldsymbol{u}^{\prime}, p^{\prime}\right)\right\rangle_{\Gamma_{L}}+\langle\boldsymbol{v}, \boldsymbol{n} \cdot \boldsymbol{\sigma}(\overline{\boldsymbol{u}}, \bar{p})\rangle_{\Gamma_{O}} \\
& +\left\langle\boldsymbol{v}, \boldsymbol{n} \cdot \boldsymbol{\sigma}\left(\boldsymbol{u}^{\prime}, p^{\prime}\right)\right\rangle_{\Gamma_{O}},
\end{aligned}
$$

which after the introduction of the symmetrization and the penalty terms for the imposition of $\overline{\boldsymbol{u}}=\boldsymbol{u}_{L}$ via Nitche's method reads,

$$
\begin{aligned}
\langle\boldsymbol{v}, \boldsymbol{n} \cdot \boldsymbol{\sigma}(\boldsymbol{u}, p)\rangle_{\Gamma} & =\langle\boldsymbol{v}, \boldsymbol{n} \cdot \boldsymbol{\sigma}(\overline{\boldsymbol{u}}, \bar{p})\rangle_{\Gamma_{L}}-\left\langle\boldsymbol{v} \cdot \boldsymbol{n}, \rho c \boldsymbol{u}^{\prime} \cdot \boldsymbol{n}\right\rangle_{\Gamma_{L}}+\left\langle\overline{\boldsymbol{u}}-\boldsymbol{u}_{L}, \boldsymbol{n} \cdot \boldsymbol{\sigma}(\boldsymbol{v}, q)\right\rangle_{\Gamma_{L}}, \\
& -\beta\left\langle\boldsymbol{v}, \overline{\boldsymbol{u}}-\boldsymbol{u}_{L}\right\rangle_{\Gamma_{L}}-\left\langle\boldsymbol{v} \cdot \boldsymbol{n}, \rho c \boldsymbol{u}^{\prime} \cdot \boldsymbol{n}\right\rangle_{\Gamma_{O}}+\left\langle\boldsymbol{v}, \boldsymbol{t}_{O}\right\rangle_{\Gamma_{O}},
\end{aligned}
$$

being $\beta$ the numerical penalty parameter and where there is no contribution over $\Gamma_{S}$, since the boundary conditions there are prescribed strongly. It just remains to provide a definition of the outflow traction $\boldsymbol{t}_{O}$. Assuming that $\Gamma_{O}$ is placed sufficiently far away from the solid boundary, i.e., in the far field-region, it is reasonable to set $\bar{p} \approx 0$ and $\boldsymbol{\nabla} \overline{\boldsymbol{u}} \approx \mathbf{0}$ 
and hence the natural condition to be imposed is $\boldsymbol{t}_{O}=\mathbf{0}$. The reader should also note that there are several terms in (12) which are known and therefore can be taken to the right hand side of the problem. Let us group those boundary terms introducing the following forms,

$$
\begin{aligned}
\mathcal{A}_{\Gamma}(\boldsymbol{U}, \boldsymbol{V}) & \doteq\left\langle\boldsymbol{v} \cdot \boldsymbol{n}, \rho c \boldsymbol{u}^{\prime} \cdot \boldsymbol{n}\right\rangle_{\Gamma_{L} \cup \Gamma_{O}}-\langle\boldsymbol{v}, \boldsymbol{n} \cdot \boldsymbol{\sigma}(\overline{\boldsymbol{u}}, \bar{p})\rangle_{\Gamma_{L}}-\langle\overline{\boldsymbol{u}}, \boldsymbol{n} \cdot \boldsymbol{\sigma}(\boldsymbol{v}, q)\rangle_{\Gamma_{L}} \\
& +\beta\langle\boldsymbol{v}, \overline{\boldsymbol{u}}\rangle_{\Gamma_{L}}, \\
\ell_{\Gamma}(\boldsymbol{V}) & \doteq \beta\left\langle\boldsymbol{v}, \boldsymbol{u}_{L}\right\rangle_{\Gamma_{L}}-\left\langle\boldsymbol{u}_{L}, \boldsymbol{n} \cdot \boldsymbol{\sigma}(\boldsymbol{v}, q)\right\rangle_{\Gamma_{L}},
\end{aligned}
$$

which will go to the left and right hand side of the problem, respectively.

Finally, the variational formulation with the boundary conditions would now read as follows: find $\boldsymbol{U} \in L^{2}\left(0, T ; \mathbb{V}^{d} \times \mathbb{Q}\right)$ such that,

$$
\left\langle\mathcal{D}_{t}(\boldsymbol{U}), \boldsymbol{V}\right\rangle+\mathcal{A}(\boldsymbol{u} ; \boldsymbol{U}, \boldsymbol{V})+\mathcal{A}_{\Gamma}(\boldsymbol{U}, \boldsymbol{V})=\mathcal{B}(\boldsymbol{V}),
$$

for all $\boldsymbol{V} \in \mathbb{V}^{d} \times \mathbb{Q}$, satisfying the initial conditions and where $\mathcal{B}(\boldsymbol{V}) \doteq \ell_{\Gamma}(\boldsymbol{V})+\langle\boldsymbol{v}, \boldsymbol{f}\rangle$.

\section{Numerical APproximation}

In this section we derive the finite element approximation of the isentropic compressible Navier-Stokes equations, which is the base of the final segregation scheme.

4.1. Galerkin finite element approximation. Let $\mathcal{T}_{h}$ be a regular-shaped and conforming partition of $\Omega$, such that $\bar{\Omega}=\cup_{\mathcal{K} \in \mathcal{T}_{h}} \mathcal{K}$. This triangulation is described by the characteristic mesh size, defined as $h \doteq \max \left\{h_{k} \mid \mathcal{K} \in \mathcal{T}_{h}(\Omega)\right\}$ with $h_{\mathcal{K}}=\operatorname{diam}(\mathcal{K})$. Let now be $\mathbb{V}_{h}^{d} \subset \mathbb{V}^{d}$ and $\mathbb{Q}_{h} \subset \mathbb{Q}$ the velocity and pressure finite element spaces associated with the triangulation. The Galerkin semi-discrete problem reads now as follows: find the pair $\boldsymbol{U}_{h} \doteq\left[\boldsymbol{u}_{h}, p_{h}\right]^{t} \in L^{2}\left(0, T ; \mathbb{V}_{h}^{d} \times \mathbb{Q}_{h}\right)$, such that:

$$
\left\langle\mathcal{D}_{t}\left(\boldsymbol{U}_{h}\right), \boldsymbol{V}_{h}\right\rangle+\mathcal{A}\left(\boldsymbol{u}_{h} ; \boldsymbol{U}_{h}, \boldsymbol{V}_{h}\right)+\mathcal{A}_{\Gamma}\left(\boldsymbol{U}_{h}, \boldsymbol{V}_{h}\right)=\mathcal{B}\left(\boldsymbol{V}_{h}\right),
$$

for all $\boldsymbol{V}_{h} \doteq\left[\boldsymbol{v}_{h}, q_{h}\right]^{t} \in \mathbb{V}_{h}^{d} \times \mathbb{Q}_{h}$, satisfying the appropriate initial conditions.

As we shall see at the end of this section, the algebraic system arising from the discrete isentropic problem is no longer of the saddle point type. This algebraic and distinctive feature indicates that the compatibility requirement on the choice of velocity and pressure interpolating spaces does not coincide with the classical inf-sup condition for incompressible flows, yet other prerequisites might be needed so as to ensure that the matrix of the whole system has a full rank. Likewise, instabilities may also arise due to the presence of the convective terms. For the sake of simplicity, and without loss of generality, we will assume for the moment that the Galerkin formulation is stable. Later, in Section 6, we will deeply describe the stabilized finite element formulation we favor, which allows one to solve highly convective cases.

4.2. Monolithic time discretization. Although in principle any time discretization method might be used, we restrict ourselves to the classical backward-difference (BDF) approximation. Let us now consider a partition of the time interval $(0, T)$ into $N$ time steps of size $\delta t$, assumed to be constant, for simplicity. Given a generic time dependent function $g(t)$ at a time step $t^{n+1}=t^{n}+\delta t$, for $n=0,1,2, \ldots$, the approximation of the time derivative of $g(t)$ of order $k=1,2, \ldots$ is written as $\delta_{k} g^{n+1} / \delta t$, where the numerator is given by the following BDF operator,

$$
\delta_{k} g^{n+1}=\frac{1}{\psi_{k}}\left(g^{n+1}-\sum_{i=0}^{k-1} \xi_{k}^{i} g^{n-i}\right),
$$

being $\psi_{k}$ and $\xi_{k}^{i}$ numerical parameters depending on the order of the temporal approximation. In particular, for the first and second order schemes, i.e. $k=1,2$, it is found 
that:

$$
\begin{aligned}
& \delta_{1} g^{n+1}=\delta g^{n+1}=g^{n+1}-g^{n}, \\
& \delta_{2} g^{n+1}=\frac{3}{2}\left(g^{n+1}-\frac{4}{3} g^{n}+\frac{1}{3} g^{n-1}\right) .
\end{aligned}
$$

In the design of fractional step schemes, it is useful to define the extrapolation operators of order $k$, formally written as $\widehat{g}_{k}^{n+1}=g^{n+1}+\mathcal{O}\left(\delta t^{k}\right)$, which for $k=1,2$ are given by,

$$
\begin{aligned}
& \widehat{g}_{1}^{n+1}=g^{n}, \\
& \widehat{g}_{2}^{n+1}=2 g^{n}-g^{n-1} .
\end{aligned}
$$

Using the aforementioned BDF schemes, the time discretization of the semi-discrete equation 15 is stated as: for $n=0,1,2, \ldots$, find $\boldsymbol{U}^{n+1} \in \mathbb{V}_{h}^{d} \times \mathbb{Q}_{h}$ such that,

$$
\left\langle\mathcal{D}_{t, k}\left(\boldsymbol{U}_{h}^{n+1}\right), \boldsymbol{V}_{h}\right\rangle+\mathcal{A}\left(\boldsymbol{u}_{h}^{n+1} ; \boldsymbol{U}_{h}^{n+1}, \boldsymbol{V}_{h}\right)+\mathcal{A}_{\Gamma}\left(\boldsymbol{U}_{h}^{n+1}, \boldsymbol{V}_{h}\right)=\mathcal{B}\left(\boldsymbol{V}_{h}\right),
$$

for all test functions. For the sake of clarity, we include also the expanded discrete problem, i.e., for $n=0,1,2, \ldots$, find $\boldsymbol{U}_{h}^{n+1} \in \mathbb{V}_{h}^{d} \times \mathbb{Q}_{h}$ such that,

$$
\begin{aligned}
& \left\langle\boldsymbol{v}_{h}, \rho^{n+1} \frac{\delta_{k} \boldsymbol{u}_{h}^{n+1}}{\delta t}\right\rangle+\left\langle\rho^{n+1} \boldsymbol{v}_{h},\left(\boldsymbol{u}_{h}^{n+1} \cdot \boldsymbol{\nabla}\right) \boldsymbol{u}_{h}^{n+1}\right\rangle+\mu\left\langle\nabla \boldsymbol{v}_{h}, \boldsymbol{\nabla} \boldsymbol{u}_{h}^{n+1}\right\rangle+\frac{\mu}{3}\left\langle\boldsymbol{\nabla} \cdot \boldsymbol{v}_{h}, \boldsymbol{\nabla} \cdot \boldsymbol{u}_{h}^{n+1}\right\rangle \\
& -\left\langle\boldsymbol{\nabla} \cdot \boldsymbol{v}_{h}, p_{h}^{n+1}\right\rangle+\left\langle\boldsymbol{v}_{h} \cdot \boldsymbol{n}, \rho^{n+1} c^{n+1} \boldsymbol{u}_{h}^{\prime n+1} \cdot \boldsymbol{n}\right\rangle_{\Gamma_{L} \cup \Gamma_{O}}+\left\langle\boldsymbol{v}_{h} \cdot \boldsymbol{n}, \bar{p}_{h}^{n+1}\right\rangle_{\Gamma_{L}} \\
& -\left\langle\boldsymbol{v}_{h}, \mu \boldsymbol{n} \cdot \nabla \overline{\boldsymbol{u}}_{h}^{n+1}\right\rangle_{\Gamma_{L}}-\frac{\mu}{3}\left\langle\boldsymbol{v}_{h} \cdot \boldsymbol{n}, \boldsymbol{\nabla} \cdot \overline{\boldsymbol{u}}_{h}^{n+1}\right\rangle_{\Gamma_{L}}+\beta\left\langle\boldsymbol{v}_{h}, \overline{\boldsymbol{u}}_{h}^{n+1}\right\rangle_{\Gamma_{L}} \\
& -\mu\left\langle\boldsymbol{n} \cdot \boldsymbol{\nabla} \boldsymbol{v}_{h}, \overline{\boldsymbol{u}}_{h}^{n+1}\right\rangle_{\Gamma_{L}}-\frac{\mu}{3}\left\langle\boldsymbol{\nabla} \cdot \boldsymbol{v}_{h}, \overline{\boldsymbol{u}}_{h}^{n+1} \cdot \boldsymbol{n}\right\rangle_{\Gamma_{L}}=\beta\left\langle\boldsymbol{v}_{h}, \boldsymbol{u}_{L}\right\rangle_{\Gamma_{L}} \\
& -\mu\left\langle\boldsymbol{n} \cdot \boldsymbol{\nabla} \boldsymbol{v}_{h}, \boldsymbol{u}_{L}\right\rangle_{\Gamma_{L}}-\frac{\mu}{3}\left\langle\boldsymbol{\nabla} \cdot \boldsymbol{v}_{h}, \boldsymbol{u}_{L} \cdot \boldsymbol{n}\right\rangle_{\Gamma_{L}}+\left\langle\boldsymbol{v}_{h}, \boldsymbol{f}\right\rangle, \\
& \left\langle q_{h}, \frac{1}{\rho^{n+1}\left(c^{2}\right)^{n+1}} \frac{\delta_{k} p_{h}^{n+1}}{\delta t}\right\rangle+\left\langle q_{h}, \frac{1}{\rho^{n+1}\left(c^{2}\right)^{n+1}} \boldsymbol{u}_{h}^{n+1} \cdot \nabla p_{h}^{n+1}\right\rangle+\left\langle q_{h}, \boldsymbol{\nabla} \cdot \boldsymbol{u}_{h}^{n+1}\right\rangle \\
& +\left\langle q_{h}, \overline{\boldsymbol{u}}_{h}^{n+1} \cdot \boldsymbol{n}\right\rangle_{\Gamma_{L}}=\left\langle q_{h}, \boldsymbol{u}_{L} \cdot \boldsymbol{n}\right\rangle_{\Gamma_{L}}
\end{aligned}
$$

for all $\boldsymbol{V}_{h} \in \mathbb{V}_{h}^{d} \times \mathbb{Q}_{h}$.

Regarding the boundary condition terms, it remains to provide a discrete expression to compute the mean components of the unknowns. At the discrete level, the time window introduced in (11) is computed as $T_{w}=N_{w} \delta t$ being $N_{w}$ a certain amount of time steps. It is proposed to use the trapezoidal rule for integration in order to compute the mean variables. The expression we use for the average values is,

$$
\overline{\boldsymbol{u}}_{h}^{n+1}=\frac{\delta t}{T_{w}}\left(\frac{1}{2} \boldsymbol{u}_{h}^{n+1}+\sum_{j=n-N_{w}+2}^{n} \boldsymbol{u}_{h}^{j}+\frac{1}{2} \boldsymbol{u}_{h}^{n-N_{w}+1}\right),
$$

and equivalently for the pressure. This expression maintains the integration implicit and second order accurate, but several time steps need to be run prior to its application so as to obtain representative data for a reliable mean computation.

4.3. Monolithic algebraic system. The fully discretized equations in (19)- 20 provide an algebraic system for the nodal values of the finite element unknowns, i.e. $\left[\boldsymbol{u}_{h}^{n+1}, p_{h}^{n+1}\right]$. 
The algebraic structure of the variational formulation in $(18)$ yields,

$$
\begin{aligned}
\mathrm{M}_{\boldsymbol{u}} \frac{\delta_{k}}{\delta t} \mathrm{U}^{n+1}+\mathrm{K}_{\boldsymbol{u}}\left(\mathrm{U}^{n+1}\right) \mathrm{U}^{n+1}+\mathrm{M}_{\Gamma} \mathrm{U}^{n+1}+\mathrm{K}_{\Gamma} \mathrm{U}^{n+1}+\mathrm{GP}^{n+1}+\mathrm{G}_{\Gamma} \mathrm{P}^{n+1} & =\mathrm{F}^{n+1}+\mathrm{F}_{\Gamma, \boldsymbol{u}}^{n+1}, \\
\mathrm{M}_{p} \frac{\delta_{k}}{\delta t} \mathrm{P}^{n+1}+\mathrm{K}_{p}\left(\mathrm{U}^{n+1}\right) \mathrm{P}^{n+1}+\mathrm{DU}^{n+1}+\mathrm{D}_{\Gamma} \mathrm{U}^{n+1} & =\mathrm{F}_{\Gamma, p}^{n+1},
\end{aligned}
$$

where subscripts $(\cdot)_{\boldsymbol{u}}$ and $(\cdot)_{p}$ refer to matrices of the momentum and continuity equation, and $\mathrm{U}$ and $\mathrm{P}$ are the arrays of nodal unknowns, velocity and pressure respectively. The dependence of matrices $\mathrm{K}_{\boldsymbol{u}}$ and $\mathrm{K}_{p}$ on the vector of velocity unknowns $\mathrm{U}$ has been explicitly displayed in order to remark the nonlinear character of the problem. In addition, $(\cdot)_{\Gamma}$ stands for terms arising from the special treatment of boundary conditions, with $M_{\Gamma}$ containing the penalty term and $\mathrm{K}_{\Gamma}, \mathrm{G}_{\Gamma}$ and $\mathrm{D}_{\Gamma}$ the remaining Nitsche and Sommerfeld contributions. It is straightforward to identify the rest of arrays in (21)-(22) and the expressions in (19)20 .

\section{Design of the Fractional Step Method}

The approach chosen in this work is to introduce a segregation technique at the pure algebraic level, as done for instance in [28] for the viscoelastic flow problem, in contrast to the space continuous level. Although specific boundary conditions were discussed for the isentropic problem in order to avoid the backscattering of sound waves, the adopted algebraic viewpoint precludes a further analysis on pressure boundary conditions for the different stages of the fractional step algorithm.

5.1. Pressure-correction algorithm. The method we propose in this section is directly linked to a pressure-correction scheme applied to the incompressible flow problem, in which a velocity guess is first computed and then corrected once the pressure is calculated. Another possibility would be to calculate first a pressure guess, what would provide a velocitycorrection-like algorithm. We will not discuss them here, yet the ideas presented next could be also used to design velocity-correction schemes (see [9] and references therein).

In order to derive the method, let us start by writing system (21)-22 in the following equivalent manner,

$$
\begin{gathered}
\mathrm{M}_{\boldsymbol{u}} \frac{\delta_{k}}{\delta_{t}} \widetilde{\mathrm{U}}^{n+1}+\mathrm{K}_{\boldsymbol{u}}\left(\widetilde{\mathrm{U}}^{n+1}\right) \widetilde{\mathrm{U}}^{n+1}+\mathrm{M}_{\Gamma} \widetilde{\mathrm{U}}^{n+1}+\mathrm{K}_{\Gamma} \widetilde{\mathrm{U}}^{n+1}+\left(\mathrm{G}+\mathrm{G}_{\Gamma}\right) \widehat{\mathrm{P}}_{k^{\prime}}^{n+1}=\mathrm{F}^{n+1}+\mathrm{F}_{\Gamma, \boldsymbol{u}}^{n+1}, \\
\frac{1}{\psi_{k} \delta t} \mathrm{M}_{\boldsymbol{u}}\left(\mathrm{U}^{n+1}-\widetilde{\mathrm{U}}^{n+1}\right)+\mathrm{N}_{\boldsymbol{u}}^{n+1}+\mathrm{N}_{\beta}^{n+1}+\mathrm{N}_{\Gamma}^{n+1} \\
+\left(\mathrm{G}+\mathrm{G}_{\Gamma}\right)\left(\mathrm{P}^{n+1}-\widehat{\mathrm{P}}_{k^{\prime}}^{n+1}\right)=0 \\
\mathrm{M}_{p} \frac{\delta_{k}}{\delta_{t}} \mathrm{P}^{n+1}+\mathrm{K}_{p}\left(\mathrm{U}^{n+1}\right) \mathrm{P}^{n+1}-\psi_{k} \delta t\left(\mathrm{D}+\mathrm{D}_{\Gamma}\right) \mathrm{M}_{\boldsymbol{u}}^{-1} \mathrm{~N}_{\boldsymbol{u}}^{n+1} \\
-\psi_{k} \delta t\left(\mathrm{D}+\mathrm{D}_{\Gamma}\right) \mathrm{M}_{\boldsymbol{u}}^{-1} \mathrm{~N}_{\beta}^{n+1}-\psi_{k} \delta t\left(\mathrm{D}+\mathrm{D}_{\Gamma}\right) \mathrm{M}_{\boldsymbol{u}}^{-1} \mathrm{~N}_{\Gamma}^{n+1} \\
-\psi_{k} \delta t\left(\mathrm{D}+\mathrm{D}_{\Gamma}\right) \mathrm{M}_{\boldsymbol{u}}^{-1}\left(\mathrm{G}+\mathrm{G}_{\Gamma}\right)\left(\mathrm{P}^{n+1}-\widehat{\mathrm{P}}_{k^{\prime}}^{n+1}\right)+\left(\mathrm{D}+\mathrm{D}_{\Gamma}\right) \widetilde{\mathrm{U}}^{n+1}=\mathrm{F}_{\Gamma, p}^{n+1}
\end{gathered}
$$

where,

$$
\begin{gathered}
\mathrm{N}_{\boldsymbol{u}}^{n+1}=\mathrm{K}_{\boldsymbol{u}}\left(\mathrm{U}^{n+1}\right) \mathrm{U}^{n+1}-\mathrm{K}_{\boldsymbol{u}}\left(\widetilde{\mathrm{U}}^{n+1}\right) \widetilde{\mathrm{U}}^{n+1} ; \mathrm{N}_{\beta}^{n+1}=\mathrm{M}_{\Gamma} \mathrm{U}^{n+1}-\mathrm{M}_{\Gamma} \widetilde{\mathrm{U}}^{n+1} ; \\
\mathrm{N}_{\Gamma}^{n+1}=\mathrm{K}_{\Gamma} \mathrm{U}^{n+1}-\mathrm{K}_{\Gamma} \widetilde{\mathrm{U}}^{n+1},
\end{gathered}
$$

and $\widetilde{U}^{n+1}$ is an auxiliary variable to which we shall refer as intermediate velocity. Likewise, $\widehat{\mathrm{P}}_{k^{\prime}}^{n+1}$ is an extrapolation of the pressure of order $k^{\prime}$ at time step $t^{n+1}$. See Equations (16)-(17) for details. The reader should note that adding up (23) and (24) we recover (21), and that 25 is obtained upon substitution into 22 of the relation between $U^{n+1}$ and 
$\widetilde{U}^{n+1}$ obtained from 24 . Generally speaking, the fractional step approach to solve the isentropic compressible Navier-Stokes problem has three steps: first compute the intermediate velocity from (23), then obtain the pressure from (25), and finally correct the velocity result using (24).

This way of proceduring allows one to segregate the calculation of the unknowns of the problem and provides a pressure-correction-like algorithm, although some extra information is needed since equations (24) and (25) still couple $\mathrm{U}^{n+1}$ and $\mathrm{P}^{n+1}$. At this point, it is very convenient to make the following observations:

Remark 5.1. One should notice that the resulting matrix from $\mathrm{DM}_{u}^{-1} \mathrm{G}$ in (25) can be viewed as an approximation to the discrete version of the Laplacian operator $\boldsymbol{\Delta}(\cdot),[29]$. In order to avoid dealing with this matrix, which has a wide stencil and might be computationally feasible only if $\mathrm{M}_{\boldsymbol{u}}$ is approximated by a diagonal matrix, we can work with $\mathrm{DM}_{\boldsymbol{u}}^{-1} \mathrm{G} \approx \mathrm{L}$ where $\mathbf{L}$ is a Laplacian matrix obtained using the gradient of the standard shape functions.

Remark 5.2. If we wanted to compute the pressure from equation (25), we would still have to face the difficulty of computing terms such as $\mathrm{D}_{\Gamma} \mathrm{M}_{\boldsymbol{u}}^{-1} \mathrm{G}, \mathrm{DM}_{\boldsymbol{u}}^{-1} \mathrm{G}_{\Gamma}$ and $\mathrm{D}_{\Gamma} \mathrm{M}_{\boldsymbol{u}}^{-1} \mathrm{G}_{\Gamma}$. Such computations can be really time consuming and burdensome. Note that an approximation similar to the one just commented above is not possible due to the character of the boundary matrices $\mathrm{D}_{\Gamma}$ and $\mathrm{G}_{\Gamma}$.

5.2. Explicit treatment of boundary terms and final segregated scheme. Having this in mind, the novel idea we propose in this work is to modify (23) and (25) in such a way that both boundary terms $\mathrm{G}_{\Gamma} \mathrm{P}^{n+1}$ and $\mathrm{D}_{\Gamma} \mathrm{U}^{n+1}$ are treated explicitly, by means of an extrapolation of the same order of the time integration scheme. This implies that system (23)- 25) would now read,

$$
\begin{gathered}
\mathrm{M}_{\boldsymbol{u}} \frac{\delta_{k}}{\delta_{t}} \widetilde{\mathrm{U}}^{n+1}+\mathrm{K}_{\boldsymbol{u}}\left(\widetilde{\mathrm{U}}^{n+1}\right) \widetilde{\mathrm{U}}^{n+1}+\mathrm{M}_{\Gamma} \widetilde{\mathrm{U}}^{n+1}+\mathrm{K}_{\Gamma} \widetilde{\mathrm{U}}^{n+1}+\mathrm{G}_{k^{\prime}}^{n+1} \\
+\mathrm{G}_{\Gamma} \widehat{\mathrm{P}}_{k}^{n+1}=\mathrm{F}^{n+1}+\mathrm{F}_{\Gamma, \boldsymbol{u}}^{n+1}, \\
\frac{1}{\psi_{k} \delta t} \mathrm{M}_{\boldsymbol{u}}\left(\mathrm{U}^{n+1}-\widetilde{\mathrm{U}}^{n+1}\right)+\mathrm{N}_{\boldsymbol{u}}^{n+1}+\mathrm{N}_{\beta}^{n+1}+\mathrm{N}_{\Gamma}^{n+1}+\mathrm{G}\left(\mathrm{P}^{n+1}-\widehat{\mathrm{P}}_{k^{\prime}}^{n+1}\right)=0, \\
\mathrm{M}_{p} \frac{\delta_{k}}{\delta_{t}} \mathrm{P}^{n+1}+\mathrm{K}_{p}\left(\mathrm{U}^{n+1}\right) \mathrm{P}^{n+1}-\psi_{k} \delta t\left(\mathrm{D}+\mathrm{D}_{\Gamma}\right) \mathrm{M}_{\boldsymbol{u}}^{-1} \mathrm{~N}_{\boldsymbol{u}}^{n+1} \\
-\psi_{k} \delta t\left(\mathrm{D}+\mathrm{D}_{\Gamma}\right) \mathrm{M}_{u}^{-1} \mathrm{~N}_{\beta}^{n+1}-\psi_{k} \delta t\left(\mathrm{D}+\mathrm{D}_{\Gamma}\right) \mathrm{M}_{\boldsymbol{u}}^{-1} \mathrm{~N}_{\Gamma}^{n+1} \\
-\psi_{k} \delta t \mathrm{DM}_{\boldsymbol{u}}^{-1} \mathrm{G}\left(\mathrm{P}^{n+1}-\widehat{\mathrm{P}}_{k^{\prime}}^{n+1}\right)+\mathrm{D} \widetilde{U}^{n+1}+\mathrm{D}_{\Gamma} \widehat{\mathrm{U}}_{k}^{n+1}=\mathrm{F}_{\Gamma, p}^{n+1} .
\end{gathered}
$$

Note that now the products $D_{\Gamma} M_{u}^{-1} G, D_{u}^{-1} G_{\Gamma}$ and $D_{\Gamma} M_{u}^{-1} G_{\Gamma}$ do not appear in the formulation and that we treat some terms on the boundary explicitly via extrapolations in $\mathrm{G}_{\Gamma} \widehat{\mathrm{P}}_{k}^{n+1}$ and $\mathrm{D}_{\Gamma} \widehat{\mathrm{U}}_{k}^{n+1}$. Formally, the splitting algorithm of order $k$ can be stated by taking $k^{\prime}=k-1$ and it entails the following steps:

(1) Compute an intermediate velocity $\widetilde{U}^{n+1}$ from 26 .

(2) Compute an approximation to the pressure $\mathrm{P}^{n+1}$ from 28 neglecting $\mathrm{N}_{\boldsymbol{u}}^{n+1}, \mathrm{~N}_{\beta}^{n+1}$ $\mathrm{N}_{\Gamma}^{n+1}$ and substituting $\mathrm{U}^{n+1}$ by $\widetilde{\mathrm{U}}^{n+1}$ in the term $\mathrm{K}_{p}\left(\mathrm{U}^{n+1}\right) \mathrm{P}^{n+1}$. It can be seen that formally this perturbation is of order $\mathcal{O}\left(\delta t^{k}\right)$. It is the key point that permits to uncouple the calculation of $\mathrm{U}^{n+1}$ and $\mathrm{P}^{n+1}$.

(3) Perform the correction and compute the end-of-step velocity $\mathrm{U}^{n+1}$ from 27) neglecting $\mathrm{N}_{u}^{n+1}, \mathrm{~N}_{\Gamma}^{n+1}$ but taking into account $\mathrm{N}_{\beta}^{n+1}$. This can be seen as a sort of Yosida factorization for the imposition of boundary conditions (see e.g. 9]).

It is well known that the extrapolation of second order of the term $\mathrm{GP}_{k^{\prime}}^{n+1}$, i.e., taking $k^{\prime}=2$, is unstable (see [9] and references inside). Hence the resulting scheme is known to be stable up to $k=2$. In fact, this issue motivated the study of velocity correction 
algorithms, which allow to design fractional step schemes of third order in time. Still, we did not observe any erratic behaviour of the term $\mathrm{G}_{\Gamma} \widehat{\mathrm{P}}_{k}^{n+1}$ for the extrapolation of second order. The final algorithm in its matrix form is included in Algorithm 1 .

$\overline{\text { Algorithm } 1 \text { First and second order fractional step scheme for the isentropic problem, }}$ $k=1,2$

(1) Nonlinear problem to compute the intermediate velocity $\widetilde{U}^{n+1}$ using the pressure extrapolations:

$\mathrm{M}_{\boldsymbol{u}} \frac{\delta_{k}}{\delta t} \widetilde{\mathrm{U}}^{n+1}+\mathrm{K}_{\boldsymbol{u}}\left(\widetilde{\mathrm{U}}^{n+1}\right) \widetilde{\mathrm{U}}^{n+1}+\mathrm{M}_{\Gamma} \widetilde{\mathrm{U}}^{n+1}+\mathrm{K}_{\Gamma} \widetilde{\mathrm{U}}^{n+1}=\mathrm{F}^{n+1}+\mathrm{F}_{\Gamma, \boldsymbol{u}}^{n+1}-\mathrm{GP}_{k-1}^{n+1}-\mathrm{G}_{\Gamma} \widehat{\mathrm{P}}_{k}^{n+1}$

(2) Compute the pressure $\mathrm{P}^{n+1}$ using the intermediate velocity from the previous step:

$\mathrm{M}_{p} \frac{\delta_{k}}{\delta t} \mathrm{P}^{n+1}+\mathrm{K}_{p}\left(\widetilde{\mathrm{U}}^{n+1}\right) \mathrm{P}^{n+1}-\psi_{k} \delta t \mathrm{LP}^{n+1}=\mathrm{F}_{\Gamma, p}^{n+1}-\mathrm{D} \widetilde{\mathrm{U}}^{n+1}-\mathrm{D}_{\Gamma} \widehat{\mathrm{U}}_{k}^{n+1}-\psi_{k} \delta t \widehat{\mathrm{L}}_{k-1}^{n+1}$

(3) Velocity correction to obtain the end-of-step velocity $U^{n+1}$ :

$$
\frac{1}{\psi_{k} \delta t} \mathrm{M}_{\boldsymbol{u}} \mathrm{U}^{n+1}+\mathrm{M}_{\Gamma} \mathrm{U}^{n+1}=\frac{1}{\psi_{k} \delta t} \mathrm{M}_{\boldsymbol{u}} \widetilde{\mathrm{U}}^{n+1}+\mathrm{M}_{\Gamma} \widetilde{\mathrm{U}}^{n+1}-\mathrm{G}\left(\mathrm{P}^{n+1}-\widehat{\mathrm{P}}_{k-1}^{n+1}\right)
$$

Although the previous technique provides a practical way to segregate the computations, some errors are introduced which can compromise the accuracy of the solution. The first error is due to the fact that the momentum equation $(26)$ is solved for the intermediate velocity $\widetilde{U}^{n+1}$ instead of the end of step velocity $\mathrm{U}^{n+1}$. On top of that, an extra Dirichlet boundary condition needs to be provided for the second step. The essential boundary condition we enforce (at the continuous level) is:

$$
\begin{aligned}
\bar{p} & =0 & & \text { in } \Gamma_{O}, \\
p^{\prime} & =\rho c\left(\boldsymbol{u}^{\prime} \cdot \boldsymbol{n}\right) & & \text { in } \Gamma_{O},
\end{aligned}
$$

where we take into account the decomposition of the pressure in average (flow) and oscillatory (acoustic) components as suggested in Section 3. Equation $(29)$ aids to enforce the NRBC when solving the continuity equation.

The inclusion of the penalization correction $\mathrm{N}_{\beta}^{n+1}$ in the last step of the algorithm aids to properly impose the boundary conditions of the problem avoiding boundary instabilities. This seems reasonable, bearing in mind that the splitting of the momentum equation in (26)-(28) needs be done taking into account boundary conditions, similarly to the case of strong imposition of boundary conditions. It is also important to note that $M_{\Gamma}$ displays a structure of mass matrix but for boundary contributions, what in turn would allow to solve directly the system for $\mathrm{U}^{n+1}$ if a lumping technique is used. Moreover, the correction of the convective term $\mathrm{N}_{\boldsymbol{u}}^{n+1}$ could also be taken into consideration in this last step, yielding a complete Yosida scheme. This would permit to derive a high order method in time (see [9], Section 4.3).

Finally, another possibility for the extrapolation of the velocity boundary term could be argued. Since $\widetilde{U}^{n+1}$ is an approximation of $\mathcal{O}\left(\delta t^{k}\right)$ to $U^{n+1}$, and the intermediate velocity is already computed when the term $\mathrm{D}_{\Gamma} \mathrm{U}^{n+1}$ needs to be treated explicitly, one could even consider to compute $\mathrm{D}_{\Gamma} \widetilde{\mathrm{U}}^{n+1}$ instead of $\mathrm{D}_{\Gamma} \widehat{\mathrm{U}}_{k}^{n+1}$. Numerical experiments show that both options provide comparable results.

\section{ISENTROPIC-COMPRESSIBLE STABILIZED FINITE ELEMENT FORMULATION}

The last part of a robust formulation consists in developing an appropriate stabilization scheme which enables to solve highly convective problems, thus avoiding spurious solutions. Apart from that, it is helpful to use formulations which permit arbitrary velocity-pressure 
interpolations, not neccessarily satisfying any inf-sup-like condition. The stabilized formulation we propose in this work is within the VMS framework and, in particular, it is based on the Orthogonal Subgrid Scale concept (see [30] for a detailed review with examples of application).

6.1. Variational Mulsti-Scale framework: The subscale concept. Let us start by considering a general transient nonlinear problem, which we will use to illustrate the VMS procedure. Hence, let us analyze,

$$
M(\boldsymbol{U}) \frac{\partial \boldsymbol{U}}{\partial t}+\mathcal{L}(\boldsymbol{U}, \boldsymbol{U})=\boldsymbol{F},
$$

being $\boldsymbol{U}$ the unknown, $M(\boldsymbol{U})$ a matrix of coefficients associated to the temporal term, $\boldsymbol{F}$ is any possible right hand side field and $\mathcal{L}(\boldsymbol{U}, \cdot)$ a spatial differential operator, linear in the second argument. The weak form of this evolution problem is formally stated as: find $\boldsymbol{U}:(0, T) \rightarrow \mathbb{W}$, such that,

$$
\left(M(\boldsymbol{U}) \frac{\partial \boldsymbol{U}}{\partial t}, \boldsymbol{V}\right)+\langle\mathcal{L}(\boldsymbol{U}, \boldsymbol{U}), \boldsymbol{V}\rangle=\langle\boldsymbol{F}, \boldsymbol{V}\rangle,
$$

for any test function $\boldsymbol{V} \in \mathbb{W}$ with appropriate regularity. Any possible terms that might arise from the imposition of boundary conditions need to be taken into account and the reader should understand that are incorporated in the duality $\langle\cdot, \cdot\rangle$.

The starting idea of the VMS methods is to consider a splitting of the space $\mathbb{W}$ of the form $\mathbb{W}=\mathbb{W}_{h} \oplus \breve{W}$, that it to say, a decomposition into a finite element part $\mathbb{W}_{h}$ and any complementary space to it $\breve{W}$, usually termed subgrid space. Such splitting induces a scale separation of unknowns and associated test functions, i.e., $\boldsymbol{U}=\boldsymbol{U}_{h}+\breve{\boldsymbol{U}}$ and $\boldsymbol{V}=\boldsymbol{V}_{h}+\breve{\boldsymbol{V}}$, such that $\boldsymbol{U}_{h}, \boldsymbol{V}_{h} \in \mathbb{W}_{h}$ and $\breve{\boldsymbol{U}}, \breve{V} \in \breve{\mathbb{W}}$. The aforementioned splitting at the continuous level of spaces, unknowns and test functions implies that one could divide (30) into two subproblems. Therefore,

$$
\begin{array}{ll}
\left(M(\boldsymbol{U}) \frac{\partial \boldsymbol{U}}{\partial t}, \boldsymbol{V}_{h}\right)+\left\langle\mathcal{L}(\boldsymbol{U}, \boldsymbol{U}), \boldsymbol{V}_{h}\right\rangle=\left\langle\boldsymbol{F}, \boldsymbol{V}_{h}\right\rangle & \forall \boldsymbol{V}_{h} \in \mathbb{W}_{h}, \\
\left(M(\boldsymbol{U}) \frac{\partial \boldsymbol{U}}{\partial t}, \breve{\boldsymbol{V}}\right)+\langle\mathcal{L}(\boldsymbol{U}, \boldsymbol{U}), \breve{\boldsymbol{V}}\rangle=\langle\boldsymbol{F}, \breve{\boldsymbol{V}}\rangle & \forall \breve{\boldsymbol{V}} \in \breve{\mathbb{W}} .
\end{array}
$$

System (31)- $(32)$ is exactly equivalent to the prior weak form $(30)$. Equation (31) is referred as the equation for the finite element unknowns whereas 32 is termed the equation for the subgrid scales.

6.2. Derivation of the subscale stabilized formulation. The objective of this technique is to define an expression to numerically compute the subscales by solving (32) so that once this is introduced into (31), we end up with only a problem for the finite element unknowns $\boldsymbol{U}_{h}$. However, several approximations have to be made since the subgrid space $\breve{W}$ is in principle infinite dimensional. Although not enough to define a numerical scheme, subcales are usually modeled as bubble functions and thus inter-element terms are neglected.

Applying integration by parts to isolate the subscale variables, Equations (31)- 32 can be rewritten as follows,

$$
\begin{aligned}
& \left(M(\boldsymbol{U}) \frac{\partial \boldsymbol{U}}{\partial t}, \boldsymbol{V}_{h}\right)+\left\langle\mathcal{L}\left(\boldsymbol{U}, \boldsymbol{U}_{h}\right), \boldsymbol{V}_{h}\right\rangle+\left\langle\breve{\boldsymbol{U}}, \mathcal{L}^{*}\left(\boldsymbol{U}, \boldsymbol{V}_{h}\right)\right\rangle=\left\langle\boldsymbol{F}, \boldsymbol{V}_{h}\right\rangle \quad \forall \boldsymbol{V}_{h} \in \mathbb{W}_{h}, \\
& \left(M(\boldsymbol{U}) \frac{\partial \boldsymbol{U}}{\partial t}, \breve{\boldsymbol{V}}\right)+\left\langle\mathcal{L}\left(\boldsymbol{U}, \boldsymbol{U}_{h}\right), \breve{\boldsymbol{V}}\right\rangle+\langle\mathcal{L}(\boldsymbol{U}, \breve{\boldsymbol{U}}), \breve{\boldsymbol{V}}\rangle=\langle\boldsymbol{F}, \breve{\boldsymbol{V}}\rangle \quad \forall \breve{\boldsymbol{V}} \in \breve{\mathbb{W}},
\end{aligned}
$$

where $\mathcal{L}^{*}(\boldsymbol{U}, \cdot)$ refers to the formal adjoint of the spatial operator $\mathcal{L}(\boldsymbol{U}, \cdot)$. Similarly as the finite element equation (31) can be understood as the projection of the original equations 
onto the finite element space, the equations for the subscales are obtained by projecting the original equations onto the corresponding space. Therefore, Equation (34) could be rewritten as,

$$
\breve{\Pi}\left[M(\boldsymbol{U}) \frac{\partial \breve{\boldsymbol{U}}}{\partial t}+\mathcal{L}(\boldsymbol{U}, \breve{\boldsymbol{U}})\right]=\breve{\Pi}\left[\mathcal{R}\left(\boldsymbol{U} ; \boldsymbol{U}_{h}\right)\right],
$$

where the symbol $\breve{\Pi}[\cdot]$ stands for the linear projection operator onto the space of subscales and $\mathcal{R}\left(\boldsymbol{U} ; \boldsymbol{U}_{h}\right) \doteq \boldsymbol{F}-\mathcal{L}\left(\boldsymbol{U}, \boldsymbol{U}_{h}\right)-M(\boldsymbol{U}) \frac{\partial \boldsymbol{U}_{h}}{\partial t}$ is identified with the finite element residual.

The additional assumption is based on replacing the spatial operator $\mathcal{L}(\boldsymbol{U}, \cdot)$ by an algebraic operator which could be computed somehow and inverted and so that the new terms have approximately the same $L^{2}$-norm as the replaced ones. This fact motivates the introduction of a matrix $\tau$, usually referred as matrix of stabilization parameters and defined element-wise in such a way that the following approximation is made,

$$
\mathcal{L}(\boldsymbol{U}, \breve{U}) \approx \boldsymbol{\tau}^{-1}(\boldsymbol{U}) \breve{U} .
$$

With the abovementioned assumption, the subscales could be computed in each element $\mathcal{K} \in \mathcal{T}_{h}(\Omega)$ by solving the following nonlinear ordinary differential equation,

$$
M(\boldsymbol{U}) \frac{\partial \breve{\boldsymbol{U}}}{\partial t}+\boldsymbol{\tau}^{-1}(\boldsymbol{U}) \breve{\boldsymbol{U}}=\breve{\Pi}\left[\boldsymbol{F}-\mathcal{L}\left(\boldsymbol{U}, \boldsymbol{U}_{h}\right)-M(\boldsymbol{U}) \frac{\partial \boldsymbol{U}_{h}}{\partial t}\right]
$$

where, for simplicity, we have assumed that the terms on the left hand side already belong to the space of subscales and thus their projection onto $\breve{\mathbb{W}}$ its equal to the terms themselves.

Different types of VMS methods arise when we provide distinct definitions of the projection operator $\breve{\Pi}$. When one considers the space of subscales as that of the residuals, i.e., one sets $\breve{\Pi}=\mathcal{I}$ (the identity operator) when applied to the finite element residuals, the method which arises is termed Algebraic SubGrid-Scale (ASGS) 31]. Although this method is the simplest one, it is not really suitable for designing fractional step schemes. The ASGS approach combined with a segregation technique involves to compute the inverse of matrices with a wide stencil, which is usually computationally unaffordable. When the space of subscales $\breve{W}$ is enforced to be $L^{2}$-orthogonal to the finite element space $\mathbb{W}_{h}$, the method is termed Orthogonal SubGrid-Scale (OSGS or simply OSS). It corresponds to taking $\breve{\Pi}=\Pi_{h}^{\perp}=\mathcal{I}-\Pi_{h}$ where $\Pi_{h}$ is the projection operator onto the finite element space without boundary conditions. This definition makes the subscales active in regions which cannot be resolved by the finite element mesh. Both of these methods are residual based by construction, and hence also consistent, being consistency understood in the finite element context, that is to say, the stabilization terms which modify the variational form of the problem vanish when the continuous solution is introduced.

6.3. Stabilized formulation applied to the isentropic Navier-Stokes problem. Now that the general procedure has been described, let us apply these ideas to the isentropic compressible case. For reasons already discussed, we consider the OSGS technique. Let us depart from the following problem for the finite element scales (still continuous in time):

$$
\begin{aligned}
&\left\langle\mathcal{D}_{t}\left(\boldsymbol{U}_{h}\right), \boldsymbol{V}_{h}\right\rangle+\mathcal{A}\left(\boldsymbol{u}^{*} ; \boldsymbol{U}_{h}, \boldsymbol{V}_{h}\right)+\mathcal{A}_{\Gamma}\left(\boldsymbol{U}_{h}, \boldsymbol{V}_{h}\right)-\sum_{\mathcal{K} \in \mathcal{T}_{h}}\left\langle\frac{1}{c^{2} \rho} \boldsymbol{u}^{*} \cdot \boldsymbol{\nabla} q_{h}+\boldsymbol{\nabla} \cdot \boldsymbol{v}_{h}, \breve{p}\right\rangle_{\mathcal{K}} \\
&-\sum_{\mathcal{K} \in \mathcal{T}_{h}}\left\langle\rho \boldsymbol{u}^{*} \cdot \boldsymbol{\nabla} \boldsymbol{v}_{h}+\mu \boldsymbol{\Delta} \boldsymbol{v}_{h}+\frac{\mu}{3} \boldsymbol{\nabla}\left(\boldsymbol{\nabla} \cdot \boldsymbol{v}_{h}\right)+\boldsymbol{\nabla} q_{h}, \breve{\boldsymbol{u}}\right\rangle_{\mathcal{K}}=\mathcal{B}\left(\boldsymbol{V}_{h}\right),
\end{aligned}
$$

for all test functions $\boldsymbol{V}_{h}$, where the symbols $\breve{\boldsymbol{u}}$ and $\breve{p}$ denote, respectively, the velocity and pressure subscales. For simplicity we will take the advection velocity to be $\boldsymbol{u}^{*}=\boldsymbol{u}_{h}$ (see e.g. 32 for the effect of taking $\boldsymbol{u}^{*}=\boldsymbol{u}_{h}+\breve{\boldsymbol{u}}$ ). A simple calculation shows that the last two 
terms on the left hand side correspond to $\left\langle\breve{U}, \mathcal{L}^{*}\left(\boldsymbol{U}, \boldsymbol{V}_{h}\right)\right\rangle$ in 33 . Likewise, the subgrid scales are computed as the solution of the following system:

$$
\begin{aligned}
\rho \frac{\partial \breve{\boldsymbol{u}}}{\partial t}+\tau_{1}^{-1} \breve{\boldsymbol{u}} & =\Pi_{h}^{\perp}\left[\boldsymbol{f}-\rho \frac{\partial \boldsymbol{u}_{h}}{\partial t}-\rho \boldsymbol{u}^{*} \cdot \boldsymbol{\nabla} \boldsymbol{u}_{h}+\mu \boldsymbol{\Delta} \boldsymbol{u}_{h}+\frac{1}{3} \mu \boldsymbol{\nabla}\left(\boldsymbol{\nabla} \cdot \boldsymbol{u}_{h}\right)-\nabla p_{h}\right] \\
\frac{1}{c^{2} \rho} \frac{\partial \breve{p}}{\partial t}+\tau_{2}^{-1} \breve{p} & =\Pi_{h}^{\perp}\left[-\frac{1}{c^{2} \rho} \frac{\partial p_{h}}{\partial t}-\frac{1}{c^{2} \rho} \boldsymbol{u}^{*} \cdot \boldsymbol{\nabla} p_{h}-\nabla \cdot \boldsymbol{u}_{h}\right]
\end{aligned}
$$

where matrix $\boldsymbol{\tau}$ in (35) has been taken of the form $\boldsymbol{\tau}=\operatorname{diag}\left(\tau_{1} \boldsymbol{I}_{d}, \tau_{2}\right), \boldsymbol{I}_{d}$ being the $d \times d$ identity matrix. Several remarks are now in order:

Remark 6.1. A simple approach is to neglect the time derivatives of the subscales in (36)(37). In this situation, the subcales are named quasi-static in contrast to dynamic subscales, when they are considered to be time-dependent. Quasi-static subscales can be unstable in time for anisotropic space-time discretization (see [33, 34, 35] and references therein).

Remark 6.2. It is assumed that the orthogonal projections of the residual temporal terms cancels, i.e., $\Pi_{h}^{\perp}\left[\rho \frac{\partial \boldsymbol{u}_{h}}{\partial t}\right] \approx 0$ and $\Pi_{h}^{\perp}\left[\frac{1}{c^{2} \rho} \frac{\partial p_{h}}{\partial t}\right] \approx 0$. They would be exactly zero if both $\rho$ and $c$ were equal to a constant, which is not the case for the isentropic problem. However, we consider as true that both variables are such that $\rho \frac{\partial \boldsymbol{u}_{h}}{\partial t}$ and $\frac{1}{c^{2} \rho} \frac{\partial p_{h}}{\partial t}$ already belong to the finite element spaces and hence its orthogonal projection onto $\mathbb{V}_{h}^{d} \times \mathbb{Q}_{h}$ vanishes. Additionally, we assume $\Pi_{h}^{\perp}[\boldsymbol{f}] \approx 0$, which yields a weakly consistent method.

From the point of view of stability, not all the terms of the finite element residual in (36)-(37) aid to enhance the stability of the formulation. Therefore, some of them could be even neglected and a less costly method emerges. Precisely, it is this last idea the one that motivates the socalled term-by-term stabilization methods ([36, 20]).

6.4. Term-by-term stabilization and monolithic formulation. The stabilization formulation we favor in this work is a term-by-term OSS approach, also referred as split OSS. Although this scheme is not completely residual-based, it has been proven that it has an optimal consistency error (see [37] for a formal discussion and numerical analysis on the Oseen equations). The key idea behind this method resides in neglecting the extra cross products among operators applied on both test and trial functions, which arise from the classical orthogonal stabilization. Let us rewrite (36)-(37) as follows,

$$
\begin{aligned}
\rho \frac{\partial \breve{\boldsymbol{u}}}{\partial t}+\tau_{1}^{-1} \breve{\boldsymbol{u}} & =-\Pi_{h}^{\perp}\left[\rho \boldsymbol{u}_{h} \cdot \boldsymbol{\nabla} \boldsymbol{u}_{h}\right]-\Pi_{h}^{\perp}\left[\boldsymbol{\nabla} p_{h}\right]+\Pi_{h}^{\perp}\left[\mu \boldsymbol{\Delta} \boldsymbol{u}_{h}\right] \\
& +\Pi_{h}^{\perp}\left[\frac{1}{3} \mu \boldsymbol{\nabla}\left(\boldsymbol{\nabla} \cdot \boldsymbol{u}_{h}\right)\right] \\
\frac{1}{c^{2} \rho} \frac{\partial \breve{p}}{\partial t}+\tau_{2}^{-1} \breve{p} & =-\Pi_{h}^{\perp}\left[\nabla \cdot \boldsymbol{u}_{h}\right]-\Pi_{h}^{\perp}\left[\frac{1}{c^{2} \rho} \boldsymbol{u}_{h} \cdot \boldsymbol{\nabla} p_{h}\right] .
\end{aligned}
$$

It is clear that omitting any of the projections in (38)-(39) would have a remarkable effect on the stability of the final formulation, yet this does not affect the general accuracy of the scheme. In order to provide stability and convergence on both convective terms, $\Pi_{h}^{\perp}\left[-\rho \boldsymbol{u}_{h} \cdot \boldsymbol{\nabla} \boldsymbol{u}_{h}\right]$ and $\Pi_{h}^{\perp}\left[-\frac{1}{c^{2} \rho} \boldsymbol{u}_{h} \cdot \nabla p_{h}\right]$ are essential. In addition, control is also needed over the pressure gradient, $\Pi_{h}^{\perp}\left[-\nabla p_{h}\right]$. Thus, we might neglect the viscous terms $\Pi_{h}^{\perp}\left[\mu \boldsymbol{\Delta} \boldsymbol{u}_{h}\right]+\Pi_{h}^{\perp}\left[\frac{1}{3} \mu \boldsymbol{\nabla}\left(\boldsymbol{\nabla} \cdot \boldsymbol{u}_{h}\right)\right]$. We also consider that both velocity and pressure subscales can be split in the form $\breve{\boldsymbol{u}}=\breve{\boldsymbol{u}}_{1}+\breve{\boldsymbol{u}}_{2}$ and $\breve{p}=\breve{p}_{1}+\breve{p}_{2}$, each component corresponding to the first and second terms in the right hand side of (38) and (39), so that the stabilization terms are independent (see [38]). 
Taking all this into account, the term-by-term finite element formulation we propose in this work reads: find $\boldsymbol{U}_{h} \in L^{2}\left(0, T ; \mathbb{V}_{h}^{d} \times \mathbb{Q}_{h}\right)$ such that,

$$
\begin{aligned}
& \left\langle\mathcal{D}_{t}\left(\boldsymbol{U}_{h}\right), \boldsymbol{V}_{h}\right\rangle+\mathcal{A}\left(\boldsymbol{u}_{h} ; \boldsymbol{U}_{h}, \boldsymbol{V}_{h}\right)+\mathcal{A}_{\Gamma}\left(\boldsymbol{U}_{h}, \boldsymbol{V}_{h}\right)-\sum_{\mathcal{K} \in \mathcal{T}_{h}}\left\langle\rho \boldsymbol{u}_{h} \cdot \nabla \boldsymbol{v}_{h}, \breve{\boldsymbol{u}}_{1}\right\rangle_{\mathcal{K}} \\
& -\sum_{\mathcal{K} \in \mathcal{T}_{h}}\left\langle\boldsymbol{\nabla} \cdot \boldsymbol{v}_{h}, \breve{p}_{1}\right\rangle_{\mathcal{K}}-\sum_{\mathcal{K} \in \mathcal{T}_{h}}\left\langle\nabla q_{h}, \breve{\boldsymbol{u}}_{2}\right\rangle_{\mathcal{K}}-\sum_{\mathcal{K} \in \mathcal{T}_{h}}\left\langle\frac{1}{c^{2} \rho} \boldsymbol{u}_{h} \cdot \nabla q_{h}, \breve{p}_{2}\right\rangle_{\mathcal{K}}=\mathcal{B}\left(\boldsymbol{V}_{h}\right),
\end{aligned}
$$

for all test functions $\boldsymbol{V}_{h} \in \mathbb{V}_{h}^{d} \times \mathbb{Q}_{h}$.

The stabilization parameters $\tau_{1}$ and $\tau_{2}$ defined over each element $\mathcal{K} \in \mathcal{T}_{h}$ contribute to provide the stabilization for the weak forms of the momentum and mass conservation equations. Their definition is based on a Fourier analysis, which we will not discuss here (see [18]). They are computed as:

$$
\tau_{1}=\left[\mathcal{C}_{1} \frac{\mu}{h^{2}}+\mathcal{C}_{2} \rho \frac{\left|\boldsymbol{u}_{h}\right| \mathcal{K}}{h}\right]^{-1} \quad ; \quad \tau_{2}=\frac{h^{2}}{\mathcal{C}_{1} \tau_{1, K}},
$$

where $\left|\boldsymbol{u}_{h}\right|_{\mathcal{K}}$ is the mean Euclidean norm of the velocity in each element $\mathcal{K} \in \mathcal{T}_{h}(\Omega)$. Note that their values are needed at each integration point. The algorithmic constants $\mathcal{C}_{1}$ and $\mathcal{C}_{2}$ depend on the polynomial order of the interpolation. For linear elements, it is commonly set $\mathcal{C}_{1}=4$ and $\mathcal{C}_{2}=2$. The four subscales $\breve{\boldsymbol{u}}_{1}, \breve{\boldsymbol{u}}_{2}, \breve{p}_{1}$ and $\breve{p}_{2}$ are computed from the solution of:

$$
\begin{array}{lll}
\rho \frac{\partial \breve{\boldsymbol{u}}_{1}}{\partial t}+\tau_{1}^{-1} \breve{\boldsymbol{u}}_{1}=-\Pi_{h}^{\perp}\left[\boldsymbol{u}_{h} \cdot \boldsymbol{\nabla} \boldsymbol{u}_{h}\right], & \frac{1}{c^{2} \rho} \frac{\partial \breve{p}_{1}}{\partial t}+\tau_{2}^{-1} \breve{p}_{1}=-\Pi_{h}^{\perp}\left[\boldsymbol{\nabla} \cdot \boldsymbol{u}_{h}\right], \\
\rho \frac{\partial \breve{\boldsymbol{u}}_{2}}{\partial t}+\tau_{1}^{-1} \breve{\boldsymbol{u}}_{2}=-\Pi_{h}^{\perp}\left[\boldsymbol{\nabla} p_{h}\right], & \frac{1}{c^{2} \rho} \frac{\partial \breve{p}_{2}}{\partial t}+\tau_{2}^{-1} \breve{p}_{2}=-\Pi_{h}^{\perp}\left[\frac{1}{c^{2} \rho} \boldsymbol{u}_{h} \cdot \nabla p_{h}\right] .
\end{array}
$$

with zero initial condition for all of them. From this point, one can choose either to include the time derivatives of the subscales or to neglect them. For the sake of clarity in the exposition, let us consider the steady behavior of subscales. Hence, $\breve{\boldsymbol{u}}_{1}=-\tau_{1} \Pi_{h}^{\perp}\left[\boldsymbol{u}_{h} \cdot \boldsymbol{\nabla} \boldsymbol{u}_{h}\right]$, $\breve{\boldsymbol{u}}_{2}=-\tau_{1} \Pi_{h}^{\perp}\left[\nabla p_{h}\right], \breve{p}_{1}=-\tau_{2} \Pi_{h}^{\perp}\left[\boldsymbol{\nabla} \cdot \boldsymbol{u}_{h}\right]$ and $\breve{p}_{2}=-\tau_{2} \Pi_{h}^{\perp}\left[\frac{1}{c^{2} \rho} \boldsymbol{u}_{h} \cdot \nabla p_{h}\right]$.

The proposed method, which replaces the weak form (15) consists in finding $\boldsymbol{U}_{h} \in$ $L^{2}\left(0, T ; \mathbb{V}_{h}^{d} \times \mathbb{Q}_{h}\right)$, such that:

$$
\begin{aligned}
\left\langle\mathcal{D}_{t}\left(\boldsymbol{U}_{h}\right), \boldsymbol{V}_{h}\right\rangle & +\mathcal{A}\left(\boldsymbol{u}_{h} ; \boldsymbol{U}_{h}, \boldsymbol{V}_{h}\right)+\mathcal{A}_{\Gamma}\left(\boldsymbol{U}_{h}, \boldsymbol{V}_{h}\right) \\
& +\mathcal{A}_{1}^{\perp}\left(\boldsymbol{u}_{h} ; \boldsymbol{U}_{h}, \boldsymbol{V}_{h}\right)+\mathcal{A}_{2}^{\perp}\left(\boldsymbol{u}_{h} ; \boldsymbol{U}_{h}, \boldsymbol{V}_{h}\right)=\mathcal{B}\left(\boldsymbol{V}_{h}\right),
\end{aligned}
$$

for all $\boldsymbol{V}_{h} \in \mathbb{V}_{h}^{d} \times \mathbb{Q}_{h}$, satisfying the proper initial conditions and where,

$$
\begin{aligned}
\mathcal{A}_{1}^{\perp}\left(\boldsymbol{u}_{h} ; \boldsymbol{U}_{h}, \boldsymbol{V}_{h}\right) & =\sum_{\mathcal{K} \in \mathcal{T}_{h}}\left\langle\rho \boldsymbol{u}_{h} \cdot \nabla \boldsymbol{v}_{h}, \tau_{1} \Pi_{h}^{\perp}\left[\rho \boldsymbol{u}_{h} \cdot \boldsymbol{\nabla} \boldsymbol{u}_{h}\right]\right\rangle_{\mathcal{K}} \\
& +\sum_{\mathcal{K} \in \mathcal{T}_{h}}\left\langle\boldsymbol{\nabla} \cdot \boldsymbol{v}_{h}, \tau_{2} \Pi_{h}^{\perp}\left[\boldsymbol{\nabla} \cdot \boldsymbol{u}_{h}\right]\right\rangle_{\mathcal{K}}, \\
\mathcal{A}_{2}^{\perp}\left(\boldsymbol{u}_{h} ; \boldsymbol{U}_{h}, \boldsymbol{V}_{h}\right) & =\sum_{\mathcal{K} \in \mathcal{T}_{h}}\left\langle\frac{1}{c^{2} \rho} \boldsymbol{u}_{h} \cdot \nabla q_{h}, \tau_{2} \Pi_{h}^{\perp}\left[\frac{1}{c^{2} \rho} \boldsymbol{u}_{h} \cdot \nabla p_{h}\right]\right\rangle_{\mathcal{K}} \\
& +\sum_{\mathcal{K} \in \mathcal{T}_{h}}\left\langle\boldsymbol{\nabla} q_{h}, \tau_{1} \Pi_{h}^{\perp}\left[\nabla p_{h}\right]\right\rangle_{\mathcal{K}} .
\end{aligned}
$$

The terms in $\mathcal{A}_{1}^{\perp}\left(\boldsymbol{u}_{h} ; \boldsymbol{U}_{h}, \boldsymbol{V}_{h}\right)$ and $\mathcal{A}_{2}^{\perp}\left(\boldsymbol{u}_{h} ; \boldsymbol{U}_{h}, \boldsymbol{V}_{h}\right)$ modify, respectively, the weak forms of the momentum and continuity equations. At the end we have obtained a stabilized formulation which adds the numerical diffusion in an efficient manner by means of completely symmetric terms. 
6.5. Algebraic formulation and stabilized fractional step algorithm. The final variational formulation of the isentropic compressible problem was stated in (40). From there, the derivation of the matrix version is straightforward and the matrix system that needs to be solved at each time step has the same algebraic structure as (21)-(22) with the addition of two stabilization matrices, namely $\mathrm{S}_{\boldsymbol{u}}$ and $\mathrm{S}_{p}$, which arise from the discretization of the stabilization forms $\mathcal{A}_{1}^{\perp}\left(\boldsymbol{u}_{h} ; \boldsymbol{U}_{h}, \boldsymbol{V}_{h}\right)$ and $\mathcal{A}_{2}^{\perp}\left(\boldsymbol{u}_{h} ; \boldsymbol{U}_{h}, \boldsymbol{V}_{h}\right)$, respectively. Note that if the time dependency of subscales is not neglected, then the right hand side vectors of the system would also be modified in order to account for the contributions of the subscales from the previous time step. With these observations in mind, the general procedure described in Section 5 is facilely extended to the stabilized algorithm.

The only nonlinear problem in the whole process is the one associated to the intermediate velocity, $\widetilde{U}^{n+1}$. We solve the nonlinearities considering a fixed-point approach, that is to say, taking the known values from the previous iteration. In Algorithm 2 we include the final scheme in its matrix form, where the superscript $i$ denotes the nonlinear iteration counter. Note that the additional stabilization term in the system for the pressure, $\mathrm{S}_{p}\left(\widetilde{\mathrm{U}}^{n+1}\right) \mathrm{P}^{n+1}$, does not need to be linearized since $\widetilde{\mathrm{U}}^{n+1}$ is already known by the time that $\mathrm{P}^{n+1}$ needs to be computed.

$\overline{\text { Algorithm } 2 \text { First and second order stabilized fractional step scheme for the isentropic }}$ problem, $k=1,2$

(1) Nonlinear problem to compute the intermediate velocity $\widetilde{U}^{n+1}$ using the pressure extrapolations:

Set $\widetilde{\mathrm{U}}^{n+1,0}=\mathrm{U}^{n}$. For $i=0,1,2, \ldots$ until convergence, compute $\widetilde{\mathrm{U}}^{n+1, i+1}$ from:

$$
\begin{aligned}
\mathrm{M}_{\boldsymbol{u}} \frac{\delta_{k}}{\delta t} \widetilde{\mathrm{U}}^{n+1, i+1}+\mathrm{K}_{\boldsymbol{u}}\left(\widetilde{\mathrm{U}}^{n+1, i}\right) \widetilde{\mathrm{U}}^{n+1, i+1} & +\mathrm{S}_{\boldsymbol{u}}\left(\widetilde{\mathrm{U}}^{n+1, i}\right) \widetilde{\mathrm{U}}^{n+1, i+1}+\mathrm{M}_{\Gamma} \widetilde{\mathrm{U}}^{n+1, i+1} \\
& +\mathrm{K}_{\Gamma} \widetilde{\mathrm{U}}^{n+1, i+1}=\mathrm{F}^{n+1}+\mathrm{F}_{\Gamma, \boldsymbol{u}}^{n+1}-\mathrm{G} \widehat{\mathrm{P}}_{\mathrm{k}-1}^{\mathrm{n}+1}-\mathrm{G}_{\Gamma} \widehat{\mathrm{P}}_{\mathrm{k}}^{\mathrm{n}+1}
\end{aligned}
$$

(2) Compute the pressure $\mathrm{P}^{n+1}$ using the intermediate velocity from the previous step:

$$
\begin{aligned}
\mathrm{M}_{p} \frac{\delta_{k}}{\delta t} \mathrm{P}^{n+1}+\mathrm{K}_{p}\left(\widetilde{\mathrm{U}}^{n+1}\right) \mathrm{P}^{n+1} & +\mathrm{S}_{p}\left(\widetilde{\mathrm{U}}^{n+1}\right) \mathrm{P}^{n+1} \\
& -\psi_{k} \delta t \mathrm{~L} \mathrm{P}^{n+1}=\mathrm{F}_{\Gamma, p}^{n+1}-\mathrm{DU}^{n+1}-\mathrm{D}_{\Gamma} \widehat{\mathrm{U}}_{k}^{n+1}+\psi_{k} \delta t \widehat{\mathrm{P}}_{k-1}^{n+1}
\end{aligned}
$$

(3) Velocity correction to obtain the end-of-step velocity $\mathrm{U}^{n+1}$ :

$$
\frac{1}{\psi_{k} \delta t} \mathrm{M}_{\boldsymbol{u}} \mathrm{U}^{n+1}+\mathrm{M}_{\Gamma} \mathrm{U}^{n+1}=\frac{1}{\psi_{k} \delta t} \mathrm{M}_{\boldsymbol{u}} \widetilde{\mathrm{U}}^{n+1}+\mathrm{M}_{\Gamma} \widetilde{\mathrm{U}}^{n+1}-\mathrm{G}\left(\mathrm{P}^{n+1}-\widehat{\mathrm{P}}_{k-1}^{n+1}\right)
$$

Finally, let us explain how we manage the orthogonal projections $\Pi_{h}^{\perp}=\mathcal{I}-\Pi_{h}$. When compared to the raw Galerkin method, the matrices emerging from the orthogonal projection of the unknowns show a wide stencil. In order to avoid dealing with them, at the $i$-th iterion of the $n$-th time step we may approximate $\Pi_{h}^{\perp}\left(g^{n, i}\right) \approx g^{n, i}-\Pi_{h}\left(g^{n, i-1}\right)$ or $\Pi_{h}^{\perp}\left(g^{n, i}\right) \approx g^{n, i}-\Pi_{h}\left(g^{n-1}\right)$ for any generic function $g$. In other words, we perform the projection by means of known values from either the previous iteration or time step. $\mathrm{Nu}-$ merical tests reveal that both options are effective, the latter being chosen in the numerical examples included next.

\section{NumericAl RESUlts}

In this section, some numerical examples are presented to show the performance of the proposed formulation. The first case we consider is a test with a manufactured solution in order to analyze the time discretization errors of the splitting technique. Later, a 2D 
low-speed viscous flow over a cylinder at $\mathrm{Ma}=0.0583$ is calculated, and finally, we include a flow around a 3D NACA 0012 airfoil at $\mathrm{Ma}=0.4$.

For all the numerical examples, the flow is considered as an ideal gas, with adiabatic coefficient $\gamma=1.4$, molar mass $\mathcal{M}=0.02897 \mathrm{~kg} / \mathrm{mol}$ and temperature $\theta_{0}=293.15 \mathrm{~K}$. Hence, the speed of sound is $c_{0}=343.29 \mathrm{~m} / \mathrm{s}$. In addition to this, the boundary formulation with the weak imposition of Dirichlet boundary conditions is used, as explained in Section 3. Hence, a penalty parameter needs to be set to perform the simulation, $\beta$. In the case of the Navier-Stokes equations, this parameters behaves as $\beta=\beta_{0}\left(\mu / h+\rho\left|\boldsymbol{u}_{h}\right|\right)$ for some constant $\beta_{0}$ and mesh size $h$, which will be fixed for each example. As previously discussed, the nonlinearities in the problem are solved via Picard's scheme. This leads to a monotonically decreasing relative error among consecutive iterations, ensuring the convergence of the method. A maximum of 20 iterations is set, and the numerical tolerance for the $L^{2}$ norm is $1 \times 10^{-6}$. In order to solve the underlying systems of linear equations, we use the Biconjugate Gradients solver, BiCGstab [39], which is already implemented in the PETSc parallel solver library [40].

7.1. A test with analytical solution. Let us first perform a simple test whose main objective is to numerically check the time convergence of the fractional step schemes defined in Algorithm 2, For this purpose we use the socalled method of manufactured solutions. In this procedure, an exact analytical solution is defined a priori and later substituted into the continuum equations in order to obtain the associated forcing terms. Continuedly, these forcing terms are introduced as perturbations in the finite element computation. The time-dependent manufactured solutions are composed of smooth functions with no physical meaning. Dirichlet boundary conditions are prescribed weakly over the boundaries upon evaluation of the velocity analytical solution and the initial conditions arise from the prescribed functions evaluated at $t=0$ over the whole computational domain.

The region we consider is the unit square, i.e. $\bar{\Omega}=[0,1] \times[0,1]$ and we assume the following manufactured fields,

$$
\begin{aligned}
& \boldsymbol{u}\left(x_{1}, x_{2}, t\right)=g(t)\left[-\cos \left(x_{1}\right) \sin \left(x_{2}\right), \sin \left(x_{1}\right) \cos \left(x_{2}\right)\right] \\
& p\left(x_{1}, x_{2}, t\right)=-\frac{1}{4} g^{2}(t)\left(\cos \left(2 x_{1}\right)+\cos \left(2 x_{2}\right)\right)
\end{aligned}
$$

with $g(t)=\sin (2 t), t \in(0,1)$ and $x_{1}$ and $x_{2}$ referring to the Cartesian axes (Figs. 2a and 2b). The values of air for density and viscosity at the bulk temperature have been used. A structured mesh of size $h=0.05$ with bilinear elements has been employed to discretize the computational domain. Finally a constant $\beta_{0}=1000$ has been chosen to ensure a proper prescription of boundary conditions, thus avoiding excessive boundary errors.

The normalized error $\mathrm{E}$ has been computed in different norms: $\ell^{\infty}\left(L^{2}(\Omega)\right.$ ) (maximum of the time sequence of spatial $L^{2}$-norms of the solution) and $\ell^{2}\left(H^{1}(\Omega)\right)\left(\ell^{2}\right.$-norm of the time sequence of spatial $H^{1}$-norms of the solution) for velocity, and $\ell^{\infty}\left(L^{2}(\Omega)\right)$ for pressure. Fig. 3a shows the convergence plot for the fractional step algorithm using a BDF1 scheme in time and Fig. 3b for the case of second order, i.e. using BDF2. The reader can note that the schemes proposed in previous sections show the desired rate of convergence, and hence the extrapolations of the boundary terms explained in Section 5 do maintain the general temporal accuracy of the method. From the convergence plots it is also observed that the spatial error is not significant for the mesh size used.

7.2. Aeolian tones of low Mach viscous flow. The second numerical example we have considered for a proper assessment of the proposed formulation is the benchmark consisting in the aerodynamic sound radiated by an uniform flow past a cylinder. This allows one to evaluate what in the literature is referred as aeolian tones of a low Mach flow (see e.g. [41]). In this example, the cylinder undergoes lift fluctuations in response to the vortex 


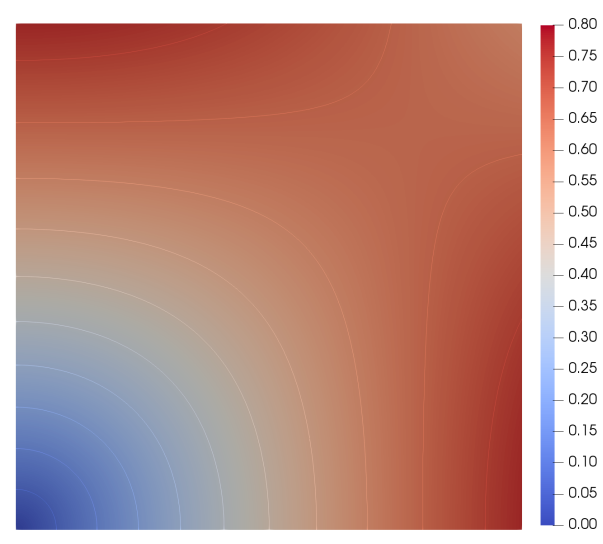

(A) Velocity, $\boldsymbol{u}\left(x_{1}, x_{2}, t=1\right)$

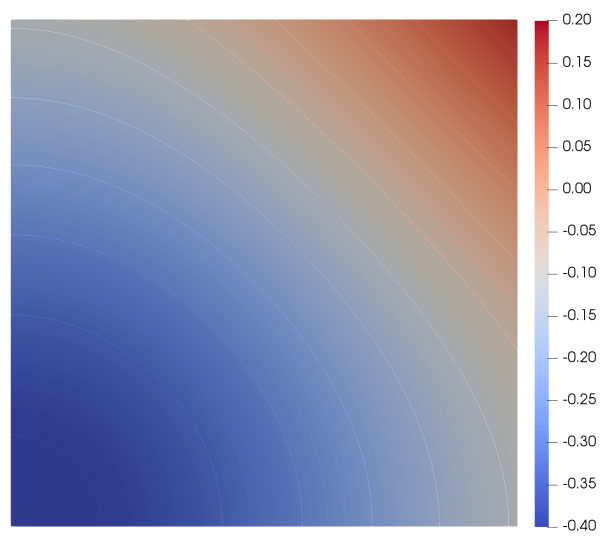

(B) Pressure, $p\left(x_{1}, x_{2}, t=1\right)$

Figure 2. Manufactured solutions (41)-42 defined for the convergence test.

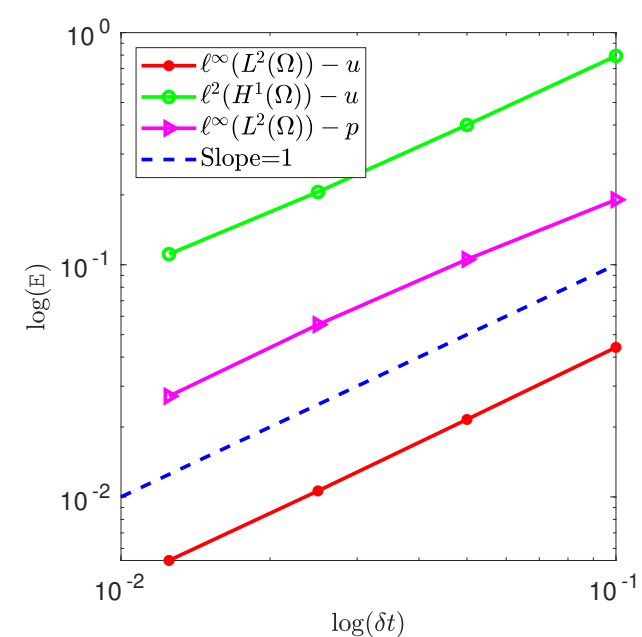

(A) BDF1

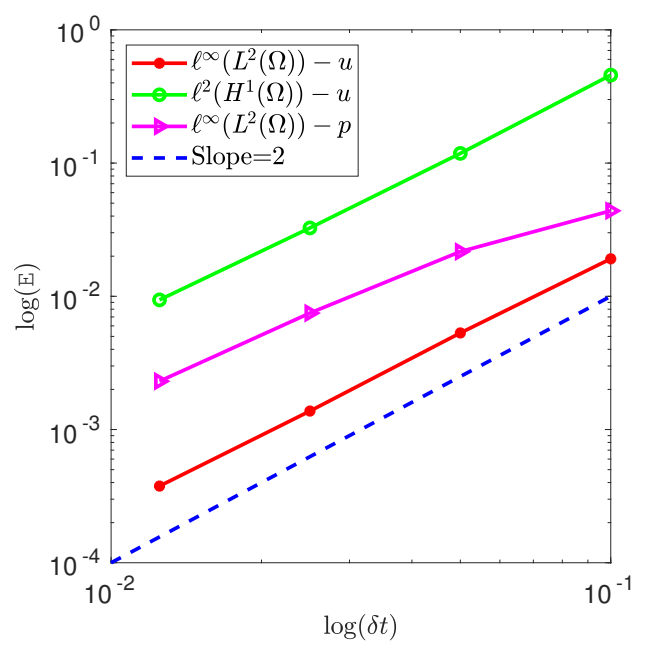

(B) BDF2

Figure 3. Convergence test results for the splitting algorithm proposed for the isentropic compressible Navier-Stokes problem.

shedding generated at the lee of the cylinder, and such fluctuations generate the sound pressure pulses. The emitted sound is named aeolian tone.

The problem domain is $[0, L] \times[0, L]$ with $L=200 \mathrm{~m}$, with the cylinder diameter $D=0.3 \mathrm{~m}$ and located at the center point of the square. The domain is big enough to describe the far field conditions far away from the cylinder. The prescription of boundary conditions is as follows: the flow is injected from the left boundary with constant horizontal velocity $U=20 \mathrm{~m} / \mathrm{s}$. Over both upper and lower walls the vertical component of the velocity is imposed to zero. These prescriptions are done weakly, using the boundary formulation previously described and with $\beta_{0}=10$. On the cylinder surface, the classical no-slip boundary condition is imposed and the outlet if left free in terms of velocity. Likewise, the Sommerfeld-like non-reflecting boundary condition is prescribed in all external boundaries in order to avoid spurious pressure reflections. We have taken a dynamic viscosity coefficient of $\mu=0.006 \mathrm{~kg} /(\mathrm{m} \mathrm{s})$ and density of $\rho=1 \mathrm{~kg} / \mathrm{m}^{3}$ to initiate the computation. All this information leads to the following Reynolds and Mach numbers, $\mathrm{Re}=1,000, \mathrm{Ma}=0.0583$, used by the benchmark solution. 
In order to complete the simulation, the filtering frequency and time window defined in (11) must be chosen small enough to allow a damping of the acoustic waves while still reproducing the flow behavior with certainty. In this sense, we set a filtering frequency of $50 \mathrm{~Hz}$ to avoid reflections at the external boundaries. The unstructured mesh for the simulation is composed of nearly 575,000 P1 elements using equal interpolation for velocity and pressure thanks to the stabilized formulation above discussed. The mesh near the cylinder wall is of $3 \times 10^{-3} \mathrm{D}$ in size, so as to capture the expected high gradients in that region. The case has been run up to $T=1 \mathrm{~s}$ with a time step size of $\delta t=1 \times 10^{-3} \mathrm{~s}$. It is important to note that the time step has to be small enough in order to be able to reproduce the aeroacoustic signal in an adequate manner. The second order BDF2 scheme has been used for the large scales time evolution, while a first order scheme has been used for the tracking of subscales. We recall here the necessity of letting the code run for several time steps prior to the application of the boundary formulation, in order to accumulate representative data for the computations. The initial condition for the simulation is provided by several time steps of an incompressible segregated solver.

In Fig. 4 we present the flow pressure contour for a certain time step of the vortex shedding cycle, qualitatively comparing the proposed fractional step scheme with its monolithic version, already validated in [6] and taken as reference. It can be observed that the pressure pulses evolve radially from the cylinder area with time, yet they do not propagate normally to the flow direction since this case is based on an uniform flow. Although some minor discrepancies might be noticed, the results are equivalent in a reasonable manner. The differences should come from the errors introduced by the splitting approach and the approximate boundary condition.

As pointed out throughout this work, the behavior of pressure waves once they reach the external artificial boundaries is a controversial situation in compressible solvers. The raw isentropic formulation would lead to the reflection of waves into the computational domain, but the compatible prescription for flow and acoustic variables adopted in this work allows the pressure pulses to abandon the domain in a smooth fashion. This fact demonstrates the satisfactory performance of the weak imposition of Dirichlet boundary conditions combined with a segregation technique and, additionally, it exposes the ability of the non-reflecting boundary conditions to attenuate the propagated sound waves.

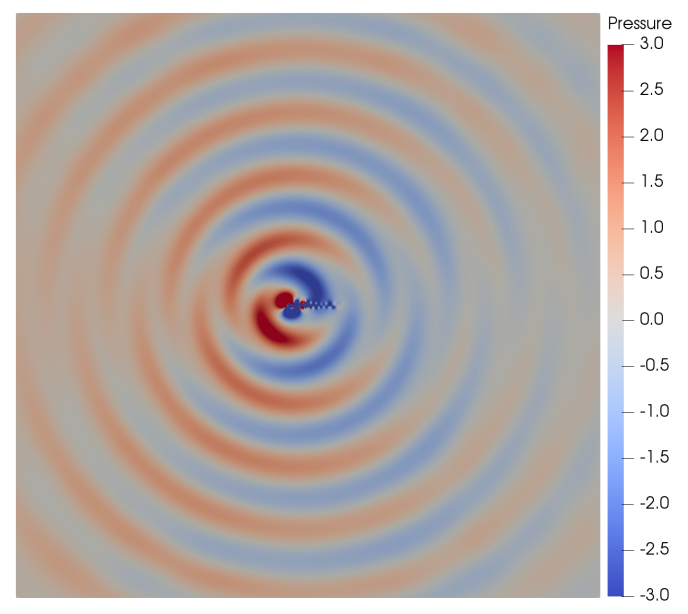

(A) Segregated approach

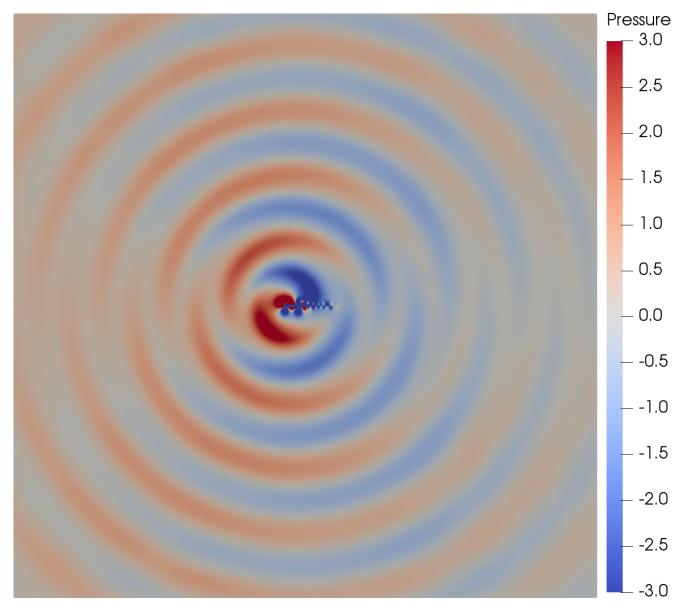

(B) Monolithic approach

Figure 4. Aeolian tones: flow pressure contour in the far field comparing the result obtained when the problem is solved using the proposed fractional step scheme and the monolithic approach in [6], taken as reference. 


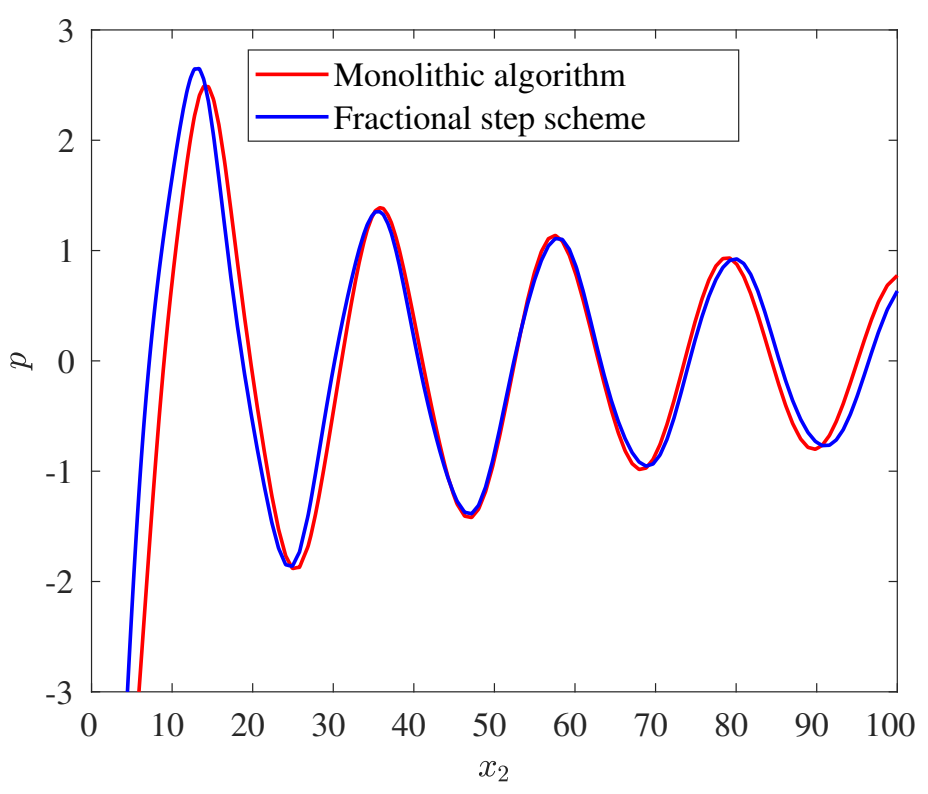

FiguRE 5. Aeolian tones: wave propagation plot containing the instantaneous pressure along the positive $x_{2}$ direction from the center of the cylinder.

In order to validate quantitatively our implementation, Fig. 5 is included, where the pressure pulse along the positive $x_{2}$ axis is depicted, for the same time instant. The reader can notice that the acoustic wave propagation obtained with the segregation algorithm reproduces the amplitude and frequency of the wave obtained with the monolithic scheme.

The computational savings that segregation techniques offer when compared to monolithic schemes are undoubted. The linear systems to be solved in fractional step methods are smaller and better conditioned, and usually each unknown requires a distinct number of iterations to solve its corresponding linear system. Although in this example we have used the same solver for all subsystems arising in the segregation method, specific solving techniques could be exploited in order to improve the performance of fractional step schemes even further. For the problem in hand, it was obtained that the CPU time of the fractional step algorithm over the CPU time of the monolithic case was 0.39 . In other words, the computational savings go up to $60 \%$.

7.3. Aerodynamic sound generated by the flow past an airfoil. The last numerical simulation we include in this section consists in the uniform flow over a NACA 0012 airfoil with an angle of attack of $5^{\circ}$. This example is intended to show the capability of the implementation to handle 3D configurations under high speed conditions. Similarly as in the previous example, the vortex shedding phenomena is at the origin of the pressure pulses which emanate from the airfoil surface.

The configuration of the domain is the box $[0, L] \times[0, H] \times[0, W]$ with $L=15 \mathrm{~m}$, $H=10 \mathrm{~m}$ and $W=1 \mathrm{~m}$. The airfoil has its trailing edge located at point $(5,5)$ and its chord line is of $d=0.1524 \mathrm{~m}$ in length. The prescription of the boundary conditions is essentially the same as for the cylinder problem in the previous subsection, yet over the walls on the $x_{3}$ direction we assign periodic boundary conditions. The incident horizontal velocity in the left surface is chosen to be $U=140 \mathrm{~m} / \mathrm{s}$, and we take $\mu=6.4 \times 10^{-5} \mathrm{~kg} /(\mathrm{m} \mathrm{s})$ and $\rho=1.2 \mathrm{~kg} / \mathrm{m}^{3}$. Therefore the Reynolds and Mach numbers are Re $\approx 400,000$ (taking the airfoil chord as characteristic length) and $\mathrm{Ma}=0.4$. The cutoff frequency for this problem is set to $1000 \mathrm{~Hz}$. 
For the simulation, the domain is discretized with a semi-structured mesh which entails about 350, 000 tetrahedral elements. Due to the highly aerodynamic character of this case, a proper meshing near the airfoil is required in order to accurately describe the velocity field in the transversal direction and hence the mean flow variables, but assessing the evolution of the boundary layer is not the goal of the present simulation. The case has been run up to $T=0.1 \mathrm{~s}$ with a time step size of $\delta t=1 \times 10^{-5} \mathrm{~s}$ departing from an initial incompressible solution in order to ease the initial convergence of the iterative solver. The coefficient for the weak imposition of Dirichlet boundary conditions has been taken as $\beta_{0}=3$. A BDF2 scheme has been used for the large scales evolution, and a first order scheme has been used for the tracking of subscales.

In Fig. 6 we include the pressure wave propagation towards the far field on a middle slice. It is observed that pressure waves evolve from the airfoil area, and eventually reach the artificial exterior boundaries. Again, the most important feature is that the solution is not polluted by spurious pressure reflections. In the previous cylinder problem, actual stagnation conditions were reached upon the consideration of a moderately big domain, obtaining an uniform pressure contour on the external boundaries. The flow perturbations only had influence over a very small near-field region in the solid surroundings, and hence the splitting of scales into mean and acoustic components was straightforward. For the present case, due to medium-subsonic Mach and high Reynolds regimes, an excessively large domain would be needed to achieve real far-field conditions. Still, our fractional step implementation is capable of prescribing the scale separation avoiding reflections in a reasonable manner, since it accounts for smooth variations of the mean flow variables which do not interfere with the acoustic field evolution.

Finally, we compare again the computational times of the segregation scheme with respect to the monolithic counterpart. For this $3 \mathrm{D}$ computation, the ratio of CPU times turns out to be 0.25 , what reveals even a further reduction in comparison with the previous $2 \mathrm{D}$ problem.

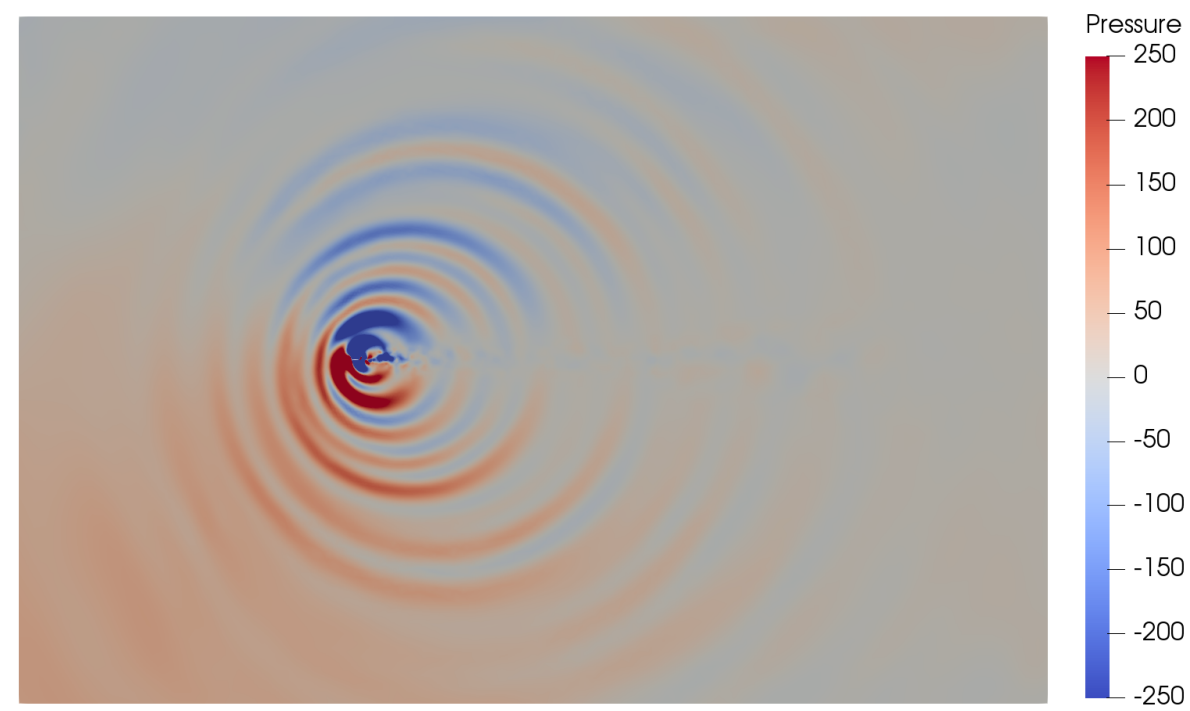

Figure 6. Airfoil: Pressure contour plot over the plane $x_{3}=0$.

\section{Conclusions}

In this article, a methodology up to second order in time to solve the isentropic compressible Navier-Stokes equations in a segregated manner has been presented. The formulation 
is constructed using the extrapolation concept at the pure algebraic level, to yield a pressure correction algorithm similar to the one commonly used for the incompressible case. From the numerical point of view, the fractional step approach has been combined with other ingredients, such as the split-orthogonal and dynamic definition of subscales, the weak imposition of Dirichlet boundary conditions via extrapolations of boundary terms and the application of non-reflecting boundary conditions, a major issue in compressible solvers.

The accuracy of the resulting schemes has been tested numerically using the method of manufactured solutions, obtaining optimal convergence rates for smooth enough solutions. Additionally, the implementation managed to reproduce the aeolian tones radiated by a flow past a cylinder, and also to complete a highly convective simulation of a 3D airfoil geometry. All these examples highlight the satisfactory performance of the proposed prescription of boundary conditions, combining Nitche's method and a Sommerfeld-like non-reflecting condition. The inclusion of the latter is crucial in this problem, in which reflections at the boundaries develop oscillations and instabilities that end up affecting the simulation results if a standard methodology is used. In addition to this, an important reduction in the CPU time with respect to the monolithic case has been verified.

The low implementation requirements when departing from a Navier-Stokes (incompressible) solver, added to the computational savings of the segregated approach, make this algorithm appealing for aeroacoustic problems for low Mach number flows, where shocks and heat transfer could be neglected.

\section{ACKNOWLEDGMEnTs}

Samuel Parada gratefully acknowledges the support received from the Agència de Gestió d'Ajust i de Recerca through the predoctoral FI grant 2019-FI-B-00607. Joan Baiges gratefully acknowledges the support of the Spanish Government through the Ramón y Cajal grant RYC-2015-17367. Ramon Codina gratefully acknowledges the support received from the ICREA Acadèmia Research Program of the Catalan Government.

\section{REFERENCES}

[1] C. Bailly, C. Bogey, and O. Marsden. Progress in direct noise computation. Noise Notes, 9(3):31-48, 2010.

[2] C. Bayona, J. Baiges, and R. Codina. Variational multi-scale finite element solution of the compressible Navier-Stokes equations. International Journal of Numerical Methods for Heat and Fluid Flow, 26:1240-1271, 2015.

[3] J. Hardin and D. Pope. An acoustic/viscous splitting technique for computational aeroacoustics. Theoretical and Computational Fluid Dynamics, 6(5-6):323-340, 1994.

[4] W. Z. Shen and J. N. Sørensen. Aeroacoustic modelling of low-speed flows. Theoretical and Computational Fluid Dynamics, 13(4):271-289, 1999.

[5] M. J. Lighthill. On sound generated aerodynamically I. General theory. Proceedings of the Royal Society - A. Mathematical, Physical and Engineering Sciences, 211(1107):564-587, 1952.

[6] A. Pont, R. Codina, J. Baiges, and O. Guasch. Unified solver for fluid dynamics and aeroacoustics in isentropic gas flows. Journal of Computational Physics, 363:11-29, 2018.

[7] A. J. Chorin. A numerical method for solving incompressible viscous problems. Journal of Computational Physics, 2:12-26, 1967.

[8] R. Teman. Sur l'approximation de la solution des equations de Navier-Stokes par la méthode des pas fractionaires (I). Archives for Rational Mechanics and Analysis, 32:135-153, 1969.

[9] S. Badia and R. Codina. Algebraic pressure segregation methods for the incompressible Navier-Stokes equations. Archives of Computational Methods in Engineering, 15(3):1-52, 2007.

[10] J. Guermond, P. Minev, and J.Shen. An overview of projection methods for incompressible flows. Computer Methods in Applied Mechanics and Engineering, 195:6011-6045, 2006.

[11] R. Codina. Pressure stability in fractional step finite element methods for incompressible flows. Journal of Computational Physics, 170:112-140, 2001. 
[12] A.N. Brooks and T.R.J. Hughes. Streamline upwind / Petrov-Galerkin formulations for convection dominated flows with particular emphasis on the incompressible Navier-Stokes equation. Computer Methods in Applied Mechanics and Engineering, 32:199-259, 1982.

[13] T.R.J. Hughes, L.P. Franca, and G.M. Hulbert. A new finite element formulation for computational fluid dynamics: VIII. The Galerkin / Least-Squares method for advective-diffusive equations. Computer Methods in Applied Mechanics and Engineering, 73:173-189, 1989.

[14] J. Donea. A Taylor-Galerkin method for convection transport problems. International Journal for Numerical Methods in Engineering, 20:101-119, 1984.

[15] T.J.R. Hughes, G.R. Feijóo, L. Mazzei, and J.B. Quincy. The variational multiscale method-a paradigm for computational mechanics. Computer Methods in Applied Mechanics and Engineering, 166:324,1998

[16] T.R.J. Hughes. Multiscale phenomena: Green's function, the Dirichlet-to-Neumann formulation, subgrid scale models, bubbles and the origins of stabilized formulations. Computer Methods in Applied Mechanics and Engineering, 127:387-401, 1995.

[17] T.J.R. Hughes, G. Scovazzi, and L.P. Franca. Multiscale and stabilized methods. Encyclopedia of Computational Mechanics, Wiley, 2004.

[18] R. Codina. Stabilized finite element approximation of transient incompressible flows using orthogonal subscales. Computer Methods in Applied Mechanics and Engineering, 191:4295-4321, 2002.

[19] E. Castillo and R. Codina. Stabilized stress-velocity-pressure finite element formulations of the NavierStokes problem for fluids with non-linear viscosity. Computer Methods in Applied Mechanics and Engineering, 279:554-578, 2014.

[20] E. Castillo and R. Codina. Finite element approximation of the viscoelastic flow problem: A nonresidual based stabilized formulation. Computer \& Fluids, 142:72-78, 2017.

[21] P. Fosso, H. Deniau, N. Lamarque, and T. Poinsot. Comparison of outflow boundary conditions for subsonic aeroacoustic simulations. International Journal of Numerical Methods in Fluids, 68(10):1207 $-1233,2012$.

[22] V. Granet, O. Vermorel, T. Léonard, I. Gicquel, and T. Poinsot. Comparison of nonreflecting outlet boundary conditions for compressible solver on unstructured grids. American Institute of Aeronautics and Astronautics, 48(10):2348 - 2364, 2010.

[23] R. Codina and J. Baiges. Weak imposition of essential boundary conditions in the finite element approximation of elliptic problems with non-matching meshes. International Journal for Numerical Methods in Engineering, 104(7):624-654, 2015.

[24] H. Espinoza, R. Codina, and S. Badia. A Sommerfeld non-reflecting boundary condition for the wave equation in mixed form. Computer Methods in Applied Mechanics and Engineering, 276:122 - 148, 2014.

[25] P.H. Oosthuizen and W.E. Carscallen. Introduction to Compressible Fluid Flow. CRC Press, 2013.

[26] M. Avila, J. Principe, and R. Codina. A finite element dynamical nonlinear subscale approximation for the low Mach number flow equations. Journal of Computational Physics, 230:7988-8009, 2011.

[27] M. Juntunen and R. Stenbeg. Nitche's method for general boundary conditions. Mathematics of Computation, 78(267):1353-1374, 2009.

[28] E. Castillo and R. Codina. First, second and third order fractional step methods for the three-field viscoelastic flow problem. Journal of Computational Physics, 296:113-137, 2015.

[29] R. Codina and A. Folch. A stabilized finite element predictor-corrector scheme for the incompressible Navier-Stokes equations using a nodal-based implementation. International Journal for Numerical Methods in Fluids, 44:483-503, 2004.

[30] R. Codina, S. Badia, J. Baiges, and J. Príncipe. Variational Multiscale Methods in Computational Fluid Dynamics. Encyclopedia of Computational Mechanics, Second Edition, pages 1-28, 2018.

[31] R. Codina. A stabilized finite element method for generalized stationary incompressible flows. Computer Methods in Applied Mechanics and Engineering, 190(20):2681-2706, 2001.

[32] O. Colomés, S. Badia, R. Codina, and J. Principe. Assessment of variational mulstiscale methods fro the large eddy simulation of turbulent incompressible flows. Computer Methods in Applied Mechanics and Engineering, 285:32-63, 2015.

[33] S. Badia and R. Codina. On a multiscale approach to the transient Stokes problem: Dynamic subscales and anisotropic space-time discretization. Applied Mathematics and Computation, 207(2):415 - 433, 2009.

[34] P.B. Bochev, M.D. Gunzburger, and R.B. Lehoucq. On stabilized finite element methods for the Stokes problem in the small time step limit. International Journal for Numerical Methods in Fluids, 53(4):573 - 597, 2007.

[35] R. Codina, J. Principe, O. Guasch, and S. Badia. Time dependent subscales in the stabilized finite element approximation of incompressible flow problem,. Computer Methods in Applied Mechanics and Engineering, 196(21):2413-2430, 2007. 
[36] T. Chacón Rebollo. A term by term stabilization algorithm for finite element solution of incompressible flow problems. Numerische Mathematik, 79:283-319, 1998.

[37] R. Codina. Analysis of a stabilized finite element approximation of the Oseen equations using orthogonal subscales. Applied Numerical Mathematics, 58:264 - 283, 2008.

[38] E. Castillo and R. Codina. Dynamic term-by-term stabilized finite element formulation using orthogonal subgrid-scales for the incompressible Navier-Stokes problem. Computer Methods in Applied Mechanics and Engineering, 349:701-721, 2019.

[39] H. A. Van der Vorst. Bi-CGSTAB: A fast and smoothly converging variant of Bi-CG for the solution of nonsymmetric linear systems. SIAM, Journal of Scientific and Statistical Computing, 13(2):631 644, 1992.

[40] S.Balay, S. Abhyankar, M. F. Adams, J. Brown, P. Brune, K. Buschelman, L. Dalcin, V. Eijkhout, W. D. Gropp, D. Kaushik, M. G. Knepley, D. A. May, L. Curfman McInnes, R. T. Mills, T. Munson, K. Rupp, P. Sanan, B. F. Smith, S. Zampini, H. Zhang, and H. Zhang. PETSc Web page. http: //www.mcs.anl.gov/petsc, 2015.

[41] O. Guasch and R. Codina. Computational aeroacoustics of viscous low speed flows using subgrid scale finite element methods. Journal of Computational Acoustics, 17(3):309 - 330, 2009. 